\title{
The McGill Planar Hydrogen Atmosphere Code (McPHAC)
}

\author{
Christian Bernt Haakonsen
}

Master of Science

Department of Physics

McGill University

Montreal,Quebec

October 21, 2009

Thesis submitted to McGill University in partial fulfillment of the requirements of the degree of Master of Science

(C) Christian Bernt Haakonsen 2009 


\section{DEDICATION}

To Rei 


\section{ACKNOWLEDGEMENTS}

I would like to thank Bob Rutledge for years of patient supervision as I grew as a scientist, and for presenting me with two interesting and challenging research problems that I have worked on over the last three years. Bob's decisiveness and clear opinions provided a valuable counter-weight to my own indecision, and I have learned a lot from him that I will benefit from in my future scientific endeavors. I would also like to thank Vicky Kaspi and Andrew Cumming for stimulating discussion on countless occasions, and for serving as role-models as researchers and educators. Finally, I would like to thank Sebastien Guillot for translating the abstract of this thesis, and for being a good friend and colleague over the last two years. 


\begin{abstract}
The McGill Planar Hydrogen Atmosphere Code v1 (McPHAC) calculates the hydrostatic equilibrium structure and emergent spectrum of a fully ionized hydrogen atmosphere in the plane-parallel approximation, at surface gravities appropriate for neutron stars. McPHAC incorporates two main improvements over previous codes for which tabulated model spectra are available: (1) Thomson scattering is treated anisotropically, which is shown to result in a $0.2-3 \%$ correction in the emergent spectral flux across the $0.1-5 \mathrm{keV}$ passband; (2) the discretization of the column depth variable in the radiative transfer problem is set independently for each discrete frequency, rather than using the same discretization as in the temperature correction procedure. Using separate discretizations for the two main components of the calculation allows the numerical uncertainty introduced in each component to be studied separately, and allows independent optimization of the two components of the calculation. Preliminary study of the numerical uncertainties in the spectra computed with McPHAC indicate that the code is capable of producing spectra with numerical uncertainties less than $0.01 \%$. Comparison with spectra from one of the previous model atmosphere codes reveals a significant deficit of flux at high energies in the spectra of the previous model, which is determined to be due to previous work not considering a sufficient number of optical depths at the highest photon frequencies. The deficit of flux in the previous model may affect the fit to the X-ray spectrum of the isolated neutron star 1RXS J185635.1-375433, and could affect the conclusion that a
\end{abstract}


hydrogen atmosphere is not an acceptable model for this source. The X-ray fitting of 1RXS J185635.1-375433 with hydrogen atmosphere models should therefore be re-visited in light of the present results. McPHAC is now released for public scrutiny and further development, as the initial step to produce unmagnetized hydrogen atmosphere emergent spectra accurate to $<0.01 \%$ in flux per energy bin. Those high-accuracy spectra are intended to be used for the study of neutron stars with modern and future X-ray spectrometers, and several future improvements to McPHAC are suggested in the present work that may be helpful in reaching the target accuracy. 


\section{RESUMÉ}

Le Code d'Atmosphère Plane d'Hydrogène de McGill (McPHAC) calcule la structure en équilibre hydrostatique et le spectre émergent d'une atmosphère d'hydrogène entièrement ionisée, avec l'approximation en plan parallèle, pour des gravités de surface comparable à celle des étoiles à neutrons. McPHAC incorpore principalement deux améliorations par rapport aux codes existants pour lesquels des spectres modélisés (sous forme de tables) sont disponibles: (1) La dispersion de Thomson est traitée de manière anisotropique, ce qui résulte en une correction de l'ordre de $0.2-3 \%$ pour le flux du spectre émergent dans la bande $0.1-5 \mathrm{keV}$. (2) La discrétisation de la variable de profondeur dans le cadre du problème de transfert radiatif est déterminée indépendamment pour chaque fréquence, au lieu d'utiliser la mme discrétisation comme cela est fait dans la procédure de correction de température. Utiliser des discrétisations séparées pour les deux principales composantes du calcul d'atmosphère permet d'étudier séparément les incertitudes numériques introduites pour chacune des composantes et permet aussi d'optimiser indépendamment les deux composantes. Des études préliminaires sur les incertitudes numériques du spectre calculé avec McPHAC indiquent que le code est capable de produire un spectre avec des incertitudes numériques de moins de $0.01 \%$. Une comparaison avec le spectre de l'un des models d'atmosphère existants révèle un déficit significatif du flux dans les hautes énergies du spectre du précèdent modèle testé. Cette différence est due au fait que le modèle précèdent ne considère pas assez de points de profondeurs dans les hautes fréquences 
des photons. Le déficit du flux observé dans le modèle précèdent peut affecter l'ajustement du spectre X de l'étoile à neutron isolée 1RXS J185635.1-375433, et peut affecter la conclusion initiale affirmant que l'atmosphère d'hydrogène n'est pas un modèle acceptable pour cette source. L'ajustement du spectre de 1RXS J185635.1-375433 avec des modèles d'atmosphère d'hydrogène devrait par conséquent tre repris, en vue des résultats présentés ici. McPHAC est désormais disponible publiquement et pour des développements futurs, en tant qu'une première étape à la production de spectre émergent d'atmosphère d'hydrogène non magnétique avec des incertitudes de flux inférieures à 0.01\% par bin d'énergie. Ces spectres de grandes précisions pourront tre utiliser pour l'étude des étoiles à neutrons avec les spectromètres X courants et futurs. Finalement, plusieurs améliorations à McPHAC, pouvant tre utiles pour atteindre la précision ciblée, sont suggérées ici. 


\section{TABLE OF CONTENTS}

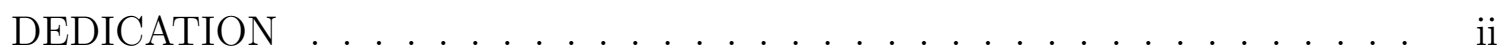

ACKNOWLEDGEMENTS ...................... iii

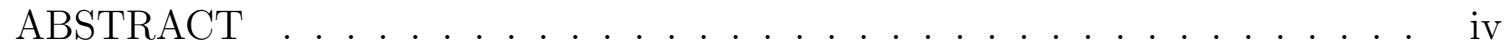

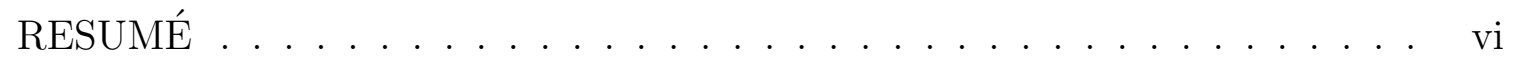

CONTRIBUTION OF AUTHORS $\ldots \ldots \ldots \ldots \ldots \ldots \ldots$

1 INTRODUCTION . . . . . . . . . . . . . . . . . 1

2 ATMOSPHERE MODEL . . . . . . . . . . . . . . . . 10

3 METHOD OF SOLUTION . . . . . . . . . . . . . . . . . 14

$3.1 \quad$ Initial Structure . . . . . . . . . . . . . . . . . . . . . . . . . 14

3.2 Radiative Transfer . . . . . . . . . . . . . . . . . . . 17

3.3 Temperature Correction . . . . . . . . . . . . . . . . . . . . 19

$4 \quad$ RESULTS . . . . . . . . . . . . . . . . . . . . . 22

$4.1 \quad$ Emergent Spectra . . . . . . . . . . . . . . . . . . 23

4.2 Anisotropic Thomson Scattering . . . . . . . . . . . . . . 24

4.3 Convergence of Computational Method . . . . . . . . . . . 25

4.4 Comparison with Previous Work . . . . . . . . . . . . . 37

4.5 Eddington Factors . . . . . . . . . . . . . . . . . . . . . 41

5 FUTURE IMPROVEMENTS . . . . . . . . . . . . . . . . . . . 45

6 DISCUSSION AND CONCLUSIONS . . . . . . . . . . . . . . 51

A RADIATIVE TRANSFER .................... 55

A.1 Basic Definitions . . . . . . . . . . . . . . 55

A.1.1 Specific Intensity _ . . . . . . . . . . . . 55

viii 
A.1.2 Flux .................... . . 56

A.1.3 Mean Specific Intensity . . . . . . . . . . . . . . . 56

A.1.4 True Absorption . . . . . . . . . . . . . . . . 56

A.1.5 Emission . . . . . . . . . . . . . . . 57

A.1.6 Thomson Scattering . . . . . . . . . . . . . . 57

A.1.7 Total Absorption Opacity . . . . . . . . . . . . . . . . 57

A.1.8 Effective Optical Depth . . . . . . . . . . . . . 58

A.1.9 Radiative Transfer Equation . . . . . . . . . . . . . . . . 58

A.1.10 Column Depth . . . . . . . . . . . . . . . . . . 59

A.1.11 Dimensionless Scattering Albedo . . . . . . . . . . . 59

A.1.12 Source Function . . . . . . . . . . . . . . . . 59

A.2 Second Order Form of the Transfer Equation . . . . . . . . . . . . 60

A.3 Computing the Specific Intensities . . . . . . . . . . . . . . 61

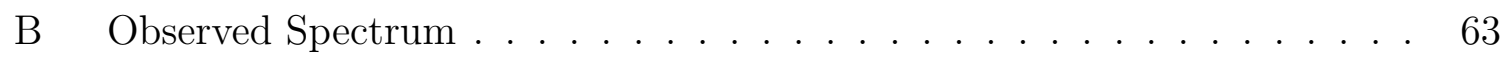

C TEMPERATURE CORRECTION SOLUTION . . . . . . . . . . 66

C.1 Temperature correction ............... 66

C.2 Discretization . . . . . . . . . . . . . . . . 68

C.3 Rybicki Method . . . . . . . . . . . . . . . . 72

C.4 Boundary Conditions ................ . 74

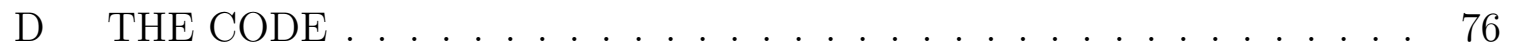

D.1 Platform and Language . . . . . . . . . . . . . . . . 76

D.2 External Routines . . . . . . . . . . . . . . . 76

D.2.1 LAPACK . . . . . . . . . . . . . . . . 77

D.2.2 BLAS . . . . . . . . . . . . . . . . 77

D.2.3 Cubic Spline Interpolation . . . . . . . . . . . . . . 77

D.2.4 Gauss-Legendre Quadrature . . . . . . . . . . . 78

D.2.5 Equation of State . . . . . . . . . . . . . 78 


\section{CONTRIBUTION OF AUTHORS}

This thesis is an adaptation to the thesis format of a paper to be submitted to the Astrophysical Journal:

- Haakonsen, C. B., Turner, M., Tacik, N., Rutledge, R. E. (2009) The McGill Planar Hydrogen Atmosphere Code (McPHAC). Astrophysical Journal, in prep.

Thus, the contributions of the co-authors should be properly acknowledged:

- Robert E. Rutledge developed the initial code that has since been de-bugged and further developed to become McPHAC v1, and started the writing of the theoretical development text that has since become the appendices of the McPHAC paper. Over the past two years, Prof. Rutledge has been involved in daily to weekly discussions on the progress of the McPHAC development, and has provided valuable guidance and insight. The introduction of the McPHAC paper has been written in close collaboration with prof. Rutledge, who has contributed approximately half the text of the introduction. For the remaining sections, prof. Rutledge has performed minor editing and some re-structuring, and has also made valuable comments regarding the figures and captions.

- Monica Turner and Nick Tacik were part of the McPHAC development process, and contributed to the text that has since become the appendices of 
the McPHAC paper. They each scrutinized large parts of the code looking for bugs, and made several improvements/fixes to the code. 


\section{CHAPTER 1 INTRODUCTION}

In recent years, there has been a large and growing interest in the application of calculated emergent spectral models to spectral observations in the optical and X-ray pass-bands.

Following the suggestion that the emergent spectrum from a quiescent transient low mass X-ray binary (qLMXB) should be expected to be an unmagnetized Hydrogen (H) atmosphere spectrum (Brown et al. 1998), H atmosphere spectra have been used to measure the radiation radii from a large number of such systems. This includes $\sim 10$ field qLMXBs, to which the distances are typically uncertain at the 25-50\% level (Rutledge et al. 1999, 2000, 2001b,a, 2002b; Wijnands et al. 2003; Tomsick et al. 2004; Campana et al. 2004; Jonker et al. 2004; Tomsick et al. 2005; in't Zand 2005; Cackett et al. 2006; Jonker et al. 2007a; Cornelisse et al. 2007; Tomsick et al. 2007; Jonker et al. 2007b; Heinke et al. 2007; Cackett et al. 2008; Degenaar et al. 2009). These modelling efforts place constraints on the 
measured radii ${ }^{1}$, and have lent support to the interpretation of deep crustal heating in neutron stars (Haensel \& Zdunik 1990; Brown et al. 1998; Haensel \& Zdunik 2008) proposed to explain their luminosities. Also, $\mathrm{H}$ atmosphere spectral results from post-outburst transients (Cackett et al. 2008, e.g.), as they cool (Ushomirsky \& Rutledge 2001; Rutledge et al. 2002c), have had significant physical implications for the reactions which take place in the neutron star crust, including neutron star core cooling rates (Yakovlev et al. 2003; Shternin et al. 2007) and neutron superfluidity in the inner crust, combined with a very low impurity parameter (i.e., a very pure crust) (Brown \& Cumming 2009).

Un-magnetized Hydrogen atmosphere models have been applied to study pulsars as well (Zavlin \& Pavlov 1998; Zavlin et al. 1998, 2002; Zavlin 2006; Bogdanov et al. 2007), usually to derive limitations on the physical sizes of their emission areas.

Hydrogen atmosphere models have been used to derive neutron star massradius constraints from X-ray observations of qLMXBs in globular clusters, where the distance uncertainties are more typically $~ 10 \%$ and lower, including in NGC 6440 (in't Zand et al. 2001); in Omega Cen (Rutledge et al. 2002a; Gendre et al. 2003b); in 47 Tuc (Heinke et al. 2003b, 2006); in NGC 6397 (Grindlay et al. 2001); in Terzan 1 (Wijnands et al. 2002); in M13 (Gendre et al. 2003a); in

${ }^{1}$ What is typically measured, is the radiation radius $R_{\infty}$, which is related to the physical radius $R$ through the neutron star mass $M$ as $R_{\infty}$ $R / \sqrt{1-(2 G M) /\left(R c^{2}\right)}$. This measurement produces a joint mass-radius constraint. 
M28 (Becker et al. 2003); A-1 in M30 (Lugger et al. 2007); in M80 (Heinke et al. 2003a); in M55 and NGC 3201 (Webb et al. 2006); in NGC 2808 (Webb \& Barret 2007); in NGC 6304 (Guillot et al. 2009a,b). These constraints are interpreted to place constraints on the dense matter equation of state - the relationship between pressure and density in non-relativistic matter at and above nuclear density. As such, these constraints are used by nuclear physicists as guidance for examining the credibility of proposed EOSs (Lattimer \& Prakash 2001, 2007). These constraints are therefore of strong observational interest in the nuclear physics community.

Hydrogen atmosphere models have been applied to isolated neutron stars (e.g., Pavlov et al. (1996); Burwitz et al. (2001); Pons et al. (2002) for 1RXS J185635.1-375433; Shevchuk et al. (2009)). In the case of 1RXS J185635.1375433 in particular, the best-fit $\mathrm{H}$ atmosphere spectrum, extrapolated to the optical passband, is significantly discrepant (by 1-2 orders of magnitude) from the observed optical flux. This discrepancy, and the absence of a natural atmospheric model which accurately describes the observed spectrum, remains one of the outstanding puzzles of neutron star atmospheres.

Thus, there are a large number of observational results, with important physics implications, which rest on the accuracy of the calculated hydrogen atmosphere models available for interpretation of observed spectra.

Most applications of hydrogen atmosphere models in X-ray spectral fitting are through tabulated models that have been made available through XSPEC (Arnaud 1996). The main such models are NSA (Zavlin et al. 1996, Z96 hereafter), 
HYD_SPECTRA (Gänsicke et al. 2002, G02 hereafter), and NSATMOS (Heinke et al. 2006, H06 hereafter). A version of the NSA model allowing for varying surface gravity, NSAGRAV, is also implemented. In addition, other non-magnetic hydrogen atmosphere models have previously been presented (e.g., Rajagopal \& Romani 1996), as well as magnetic hydrogen atmosphere models that can be run in the zero field limit (e.g., Lloyd 2003), but these will not be considered in depth in the present work.

The three main non-magnetic hydrogen atmosphere models (Z96; G02; H06) have many similarities in the assumptions made and in the method of solution. Common to all three models are the assumptions of a plane-parallel atmosphere, isotropic and coherent unpolarized scattering, local thermodynamic equilibrium (LTE), radiative equilibrium, and hydrostatic equilibrium. Additional assumptions common to one or more models are complete ionization (H06), ideal equation of state (H06), neglecting radiation pressure (Z96; G02), neglecting self-irradiation (Z96; G02), and neglecting electron conduction (Z96). The form of the radiative transfer equation solved in each of the three models is the same, including both a true absorption opacity and a scattering opacity. The calculation of the opacities, however, differs in the three models: In the first model (Z96), occupation probability formalism (Zavlin et al. 1994) is used to calculate the true absorption opacity, while the uncorrected Thomson scattering opacity is used ${ }^{2}$.

\footnotetext{
${ }^{2}$ No explicit statement is made in the paper (Z96) about any corrections to the Thomson scattering opacity.
} 
In the second model (G02), OPAL data (Iglesias \& Rogers 1996) are used for the total (true absorption plus scattering) opacity, and the Thomson scattering opacity is corrected for collective effects (Boercker 1987). In the third model (H06), uncorrected thermal free-free true absorption opacity and uncorrected Thomson scattering opacity are used ${ }^{3}$. All other differences between the three models relate to the way the coupled atmosphere structure and radiative transfer equations are solved, but these differences are not expected to lead to quantitative differences in the resulting spectra as long as adequate levels of discretization and iteration are used in each model.

Non-magnetic atmosphere models also exist for a variety of elements and mixtures (e.g., Z96; Rajagopal \& Romani (1996); G02), but only the resulting tabulated spectra, and not the code used to calculate them, have been made available for critical inspection. Discrepancies in the spectra produced by different groups have in some cases led to the discovery of coding bugs (cf. Pons et al. (2002) for discussion of the discovery of such a bug, found in Fe atmospheres calculated by earlier work, which came to light only through comparison with other calculations).

Despite their wide use in astrophysics, the uncertainties on the available tabulated spectra are poorly quantified, and these uncertainties are not considered in observational analysis; the uncertainties are typically assumed to be negligible

\footnotetext{
${ }^{3}$ No explicit statement is made in the paper (H06) about the detailed form used for these opacities.
} 
( $\lesssim 1 \%$ in spectral intensity, as a function of photon energy). However, to produce reliable mass-radius constraints from existing high-quality X-ray spectra, and even higher quality spectra which will be available from future X-ray missions such as the proposed International X-ray Observatory, model $\mathrm{H}$ atmosphere spectra with quantified uncertainties in each spectral bin are required. In particular, simultaneous constraints on the mass and radius of a neutron star at the $5 \%$ level will require statistical uncertainties in each bin of the observed spectrum of better than $0.1 \%$ (R.E. Rutledge, private comm.), so the model spectra should have uncertainties well below $0.1 \%$ for these to be negligible compared to the statistical uncertainty on the observed spectrum. Thus, an appropriate target accuracy for future model $\mathrm{H}$ atmosphere spectra aimed at producing simultaneous mass and radius constraints at the $5 \%$ level is $0.01 \%$ in each spectral bin.

The descriptions of the existing hydrogen atmosphere models (NSATMOS, NSA, NSAGRAV, and HYD_SPECTRA) which are present in the literature, provide little guidance on the uncertainties in their resulting spectra. For example, NSA (Z96) states that the calculation was permitted to proceed until the effective temperature in the emergent spectrum was within $<1 \%$ of the target effective temperature. However, this offers no reproducible constraint on the uncertainty in the emergent spectral intensity at a given photon energy; it may well be that the code implementation produces an underestimate of the intensity near the peak of the spectrum far in excess of $1 \%$, and a compensating overestimate of the intensity in the wings, or vice versa. Since the codes are not public, this cannot be investigated by critical researchers. 
Common to all these models, is that only the resulting spectra, and not the underlying coded algorithms, have been made available for inspection and critical assessment to the scientific community. Thus, though the model assumptions and basic equations are described in the corresponding papers and cited references, the implemented method for numerical solution of those equations is not available for scrutiny.

Moreover, simple ideas for improvements or further investigation held by other scientists are unlikely to be followed up, since the authors of the original code may not be motivated to implement such improvements, and since the amount of work involved in independently developing similar code is too large to justify pursuing a simple variation on something that has already been done.

The present work addresses the issue of spectral uncertainties in two ways. First, the numerical convergence of the current method is discussed at length in Section 4.3. Second, the source code is made available to the community, so that the uncertainties for specific parameter ranges, or due to the various inputs, can be probed by researches who wish to apply these models. The authors expect that numerical and model uncertainties will receive more attention in future codepresentation papers, and that other source-codes be made open-source, such that more meaningful and extensive comparisons can be made between models/codes.

The availability of the source code allows researchers to investigate the effects of any of the parameters or inputs of the code on the spectra, and to investigate the effect of relaxing certain model assumptions by generalizing the code. This 
cannot be done with the existing $\mathrm{H}$ atmosphere spectral results (Z96, G02, H06), because the code is not available to do so.

Open source code addresses an important issue in modern science, as has recently been discussed in the context of coming large projects (Weiner et al. 2009). Large private source codes cannot be scrutinized by the community, and the large complexity often deters other groups from independently reproducing the results. Thus, though the theoretical development is scrutinized in the peerreview process, the implementation is not, and coding bugs that significantly affect the results can go unnoticed for extended periods of time. One example is the previously mentioned bug in the Fe atmospheres of a previous work (cf. Pons et al. 2002).

This thesis marks the beginning of an effort to produce non-magnetic hydrogen atmosphere model spectra, for comparison with astrophysical data, accurate to $0.01 \%$ in each spectral intensity bin (to allow simultaneous constraints on the mass and radius of a neutron star at the $5 \%$ level). Many computational and physical aspects of atmospheric modelling must be carefully considered to achieve this goal, and many improvements to the open-source code presented here are needed before spectra of this accuracy and confidence can be produced.

This thesis and the associated open-source code (McPHAC) presents the analytic equations of radiative transfer and atmospheric structure, their discretization, and the complete details of the calculation of an emergent intensity spectrum, dependent upon the neutron star mass and radius, and the effective temperature. The organization of this thesis is as follows. In Chapter 2, the basic atmospheric 
model is described. The method of solution of the equations of radiative transfer and stellar structure is described in Chapter 3. In Chapter 4, there is discussion of some of the results of the calculation, including its convergence as a function of the depth and angular discretization, the effect of treating Thompson scattering anisotropically, and a comparison of the resulting spectra with one of the widely used existing spectral models (Z96). In Chapter 5, a number of possible improvements to the code are discussed, which may be necessary to ensure that the accuracy in the emergent spectra improves from the present approximate value ( $\sim$ few percent in spectral intensity as a function of photon energy) to a goal value of $0.01 \%$. In Chapter 6, the improvements offered by the present work, and main results, are summarized. 


\section{CHAPTER 2 ATMOSPHERE MODEL}

This section presents a brief review of the atmosphere model used in the McPHAC code.

Many of the assumptions adopted in previous hydrogen atmosphere models are also made in the present work:

1. magnetic fields are neglected, limiting the validity of the computed spectra to atmospheres where $B \ll 10^{10}\left(T / 10^{6} K\right) G(\mathrm{Z} 96)$;

2. complete ionization is assumed, which is only a good approximation for $T_{\text {eff }} \gtrsim 3 \times 10^{5} \mathrm{~K}(\mathrm{H} 06) ;$

3. coherent scattering is assumed, which could lead to inaccuracies in the spectra at energies greater than $5 \mathrm{keV}$ (one percent of the electron rest mass);

4. hydrostatic and radiative equilibrium;

5. no electron conduction;

6. self irradiation is ignored, limiting accuracy for neutron stars with $R<$ $(3 / 2) R_{S}(\mathrm{H} 06) ;$

7. the plane parallel assumption is used, since the scale height of the atmosphere is much smaller than the radius of the neutron star;

8. radiation pressure is neglected, which is appropriate for atmospheres with $T_{\text {eff }} \ll 10^{7} \mathrm{~K}(\mathrm{Z} 96)$. 
Following these assumptions, a standard plane-parallel atmosphere model is used in conjunction with a radiative transfer calculation. A summary of the literature used is given in the following more completely described approach. The atmospheric modelling and radiative transfer follows the descriptions elsewhere (Mihalas 1970, 1978; Rybicki \& Lightman 1979), and benefitted greatly from the discussions of previous calculations (Z96, G02, H06). A pedagogical review of radiative transfer, which includes definitions of terms used throughout this work, is given in Appendix A.

Neglecting radiation pressure, the pressure at any depth is equal to the weight of the integrated column $y$ of material above it:

$$
P(y)=g_{s} y,
$$

where $g_{s}$ is the surface gravity, taken to be uniformly constant as per the planeparallel atmosphere assumption. The surface gravity is assumed not to vary throughout the atmosphere since a negligible fraction of the neutron star mass is contained in the atmosphere, and its thickness is negligible compared to the neutron star radius.

The pressure is related to the temperature $T$ and density $\rho$ through the equation of state (EOS), $P=P(\rho, T)$, which can be used to find the density at a given pressure (i.e. column depth) and temperature. To solve for the structure of the atmosphere, one additional expression is needed: the temperature as a function of column depth $T=T(y)$, which is referred to as the temperature profile. The temperature profile depends on the radiative transfer through the atmosphere. 
Thus, the temperature profile, atmospheric structure and radiative transfer must be solved for jointly and iteratively.

Setting up the standard Feautrier problem (Feautrier 1964, cf. Appendix A), the second order form of the radiative transfer equation is

$$
\mu^{2} \frac{d^{2} P_{\nu}(y, \mu)}{d \tau_{\nu}}=P_{\nu}(y, \mu)-S_{\nu}(y, \mu)
$$

where $P_{\nu}$ (cf.Equation A.16) is the average of the ingoing and outgoing specific intensities in a given direction, $S_{\nu}\left(\tau_{\nu}, P_{\nu}\right)$ is the source function in that direction, and $\mu$ is the cosine of the angle to the outward normal. The boundary conditions used are zero incident specific intensity at the surface $\left(P_{\nu}\right.$ is half the outgoing specific intensity at the surface), and $P_{\nu}$ equal to the blackbody intensity at the deepest point considered. In terms of the true absorption opacity $\kappa_{\nu}$, and the reduced scattering opacity $\sigma_{\mathrm{T}, \mathrm{r}}$, the source function can be expressed as

$$
S_{\nu}(y, \mu)=\frac{\kappa_{\nu}}{\kappa_{\nu}+\sigma_{\mathrm{T}, \mathrm{r}}} B_{\nu}(T(y))+\frac{2}{\kappa_{\nu}+\sigma_{\mathrm{T}, \mathrm{r}}} \int_{0}^{1} P_{\nu}\left(y, \mu^{\prime}\right) \frac{d \sigma_{\mathrm{T}, \mathrm{r}}}{d \mu^{\prime}}\left(\mu, \mu^{\prime}\right) d \mu^{\prime},
$$

where $d \sigma_{\mathrm{T}, \mathrm{r}} / d \mu^{\prime}\left(\mu, \mu^{\prime}\right)$ is the differential amount of scattering from directions $\mu^{\prime}$ into directions $\mu$. Note that the present form of the source function differs from that used in previous works (Z96; G02; H06) since the scattering is not assumed to be isotropic. The source function depends on the temperature profile of the atmosphere, so the coupled radiative transfer and structure equations must be solved to calculate the emergent spectrum.

For a neutron star with with a homogeneous temperature distribution across the surface, the observed flux at a distance $D$ is related to the emergent flux 
$F(R, \nu)$, where $R$ is the neutron star radius, through the relation (McClintock et al. 2004)

$$
F\left(D, \nu_{\mathrm{obs}}\right)=(1+z)^{-1} \frac{R^{2}}{D^{2}} F\left(R,(1+z) \nu_{\mathrm{obs}}\right)
$$

where $\nu_{\mathrm{obs}}$ is the observed frequency. The redshift $z$ is defined through

$$
1+z=\frac{1}{\sqrt{1-\frac{2 G M}{R c^{2}}}}
$$

where $G$ is Newton's constant, and $M$ is the mass of the neutron star. The derivation of this expression has been given previously (McClintock et al. 2004), but for clarity an alternate derivation is given in Appendix B. 


\section{CHAPTER 3 METHOD OF SOLUTION}

To solve the coupled radiative transfer and structure equations, an iterative scheme is adopted, similar to that used in a previous model (Z96). An initial guess at the temperature profile is required to calculate the initial radiative transfer, the solution to which is then used to calculate a correction to the temperature profile. The radiative transfer is then re-calculated based on the corrected temperature profile, which allows a further correction to the temperature profile to be calculated. Iteration proceeds until the maximum fractional temperature correction computed for any depth falls below a chosen threshold $\left(10^{-4}\right.$ throughout the present work).

\subsection{Initial Structure}

The main inputs to the model are the target effective temperature, $T_{\text {eff }}$, and the surface gravity $g_{s}$. The variable $\nu$ is discretized into $K$ frequencies, logarithmically spaced between some minimum and maximum multiple of $k_{B} T_{\text {eff }}$ (0.05 and 120, respectively, throughout the present work). The variable $y$ is discretized into $N$ column depths, logarithmically spaced between a minimum and maximum column depth. If the largest column depth corresponds to less than a specified minimum number of optical depths at the largest frequency considered (minimum 80 optical depths are required throughout the present work, except where specified in Section 4.4), the maximum column depth is doubled until more 
than the minimum number of optical depths are considered. The variable $\mu$ is discretized into $M$ angles, based on a Gauss-Legendre quadrature scheme.

The pressure at each column depth can be computed using Equation 2.1. In the Eddington approximation, the temperature profile satisfies the equation (Z96)

$$
\frac{d}{d y} \frac{T}{T_{\mathrm{eff}}}=\frac{3}{16} k_{\mathrm{R}}\left(\frac{T}{T_{\mathrm{eff}}}\right)^{3},
$$

where $k_{\mathrm{R}}$ it the Rosseland mean total absorption opacity. This equation can be integrated from the surface to get the initial temperature profile, using the boundary condition $T(0)=0.265 T_{\text {eff }}$. This boundary condition is found to be closer to the final converged value for hydrogen atmospheres than that used for this and other compositions in previous work (Z96). At each step in the integration, $\rho$ is computed using OPAL EOS data (Rogers et al. 1996), and the Rosseland mean opacity $k_{\mathrm{R}}$ is computed from $\kappa_{\nu}$ and $\sigma_{\mathrm{T}, \mathrm{r}}$.

The specific forms of the opacities used are the reduced Thomson scattering opacity, $\sigma_{\mathrm{T}, \mathrm{r}}=\sigma_{T} /\left(m_{p}+m_{e}\right)$, and the free-free true absorption opacity (Rybicki \& Lightman 1979) ${ }^{1}$

$$
\kappa_{\nu}=\frac{4 e^{6}}{3 m_{e} h c} \sqrt{\frac{2 \pi}{3 k m_{e}}} T^{-1 / 2} \frac{\rho}{\left(m_{p}+m_{e}\right)^{2}} \nu^{-3}\left(1-e^{-h \nu / k T}\right) \bar{g}_{f f} \mathrm{~cm}^{2} \mathrm{~g}^{-1}
$$

where $\bar{g}_{f f}$ is the temperature-averaged free-free Gaunt factor, and all units are in cgs.

\footnotetext{
${ }^{1}$ Note that the notation $\alpha_{\nu}^{\mathrm{ff}}$ used on page 162 of the reference corresponds to $\rho \kappa_{\nu}$ in the present work.
} 
The temperature-averaged free-free Gaunt factor is commonly expressed in terms of the scaled frequency $u=h \nu / k T$ and scaled temperature $\gamma^{2}=Z^{2} \mathrm{Ry} /(k T)$, and is available in tabulated form (Sutherland 1998) ${ }^{2}$. At present, a 2D linear interpolation of this data is performed to find the needed Gaunt factors, but this could be upgraded to a more accurate spline procedure. The tabulated data are available for $u$ and $\gamma^{2}$ values in the range $10^{-4}$ to $10^{4}$. In principle it is possible to specify parameters that lead to $u$ values outside this range in certain regions of the atmosphere. However, those regions are likely to be very deep in the atmosphere for the lowest frequencies, or near the surface at the highest frequencies. Low frequencies carry practically no flux deep in the atmosphere, while the upper layers of the atmosphere are transparent to high frequency photons, so the Gaunt factor will not be significant in these regions. Thus, an adequate solution is to return

2 The table was downloaded from http://www.mso.anu.edu.au/ ralph/ data/freefree/gffgu. dat (last updated 2000 March 6). However, it appears that the column labels $u$ and $\gamma^{2}$ are reversed, both in the online table and in the corresponding table in the paper, compared to what they should be. The data in the table only matches the corresponding figure in the same paper if the labels are reversed. That figure appear to be in agreement with previous work (Karzas \& Latter 1961), so it is most likely the tables that are mislabeled, rather than the figure. Several attempts to get a comment on this issue form the author of the paper have failed, but two separate mentions of this issue appear elsewhere (Ogley et al. 2002, and http://hea-www.harvard.edu/PINTofALE/pro/external/sutherland_ch.pro), so the columns are assumed to have been mislabeled when reading the tabulated data. 
the closest value on the boundary of the grid if a Gaunt factor is requested for parameters outside the grid.

\subsection{Radiative Transfer}

Having obtained an initial (or later, a corrected) atmospheric structure, the radiative transfer through the atmosphere must now be solved. The assumption of coherent scattering leads to an important computational simplification, in that the radiative transfer equation for a fixed atmospheric structure can be solved for one frequency at a time, since there is no direct frequency coupling in Equations 2.2 and 2.3. The only frequency coupling in the radiative transfer is through the effect of radiative transfer on the structure of the atmosphere, which will enter into the temperature correction procedure.

At a given frequency, there will be some maximum optical depth from beyond which there is not a significant fractional contribution to the flux at the surface. Thus, to calculate the flux at the surface, it is sufficient to consider column depths down to some maximum optical depth at that frequency. The maximum optical depth needed for a specific fractional flux contribution at the surface will vary with the frequency considered and the structure of the atmosphere, but in the present work a single pre-specified maximum number of optical depths is used at each frequency (80 optical depths are used throughout the present work).

For each frequency, the column depth is discretized into $N_{\text {rad }}$ depth points between the minimum column depth and the first column depth corresponding to more than the specified maximum number of optical depths. The temperature profile is then interpolated to these depth points, and the density and opacities 
computed there. The radiative transfer problem must then be solved on this frequency specific grid of column depths and angles.

For the fixed atmospheric structure, the radiative transfer equation (Equation 2.2, with the specific source function given in Equation 2.3) is solved using a hermitian method described completely in previous work (Auer 1976). The general form of the transfer equation solved in that work is

$$
\mu_{j}^{2} \frac{d^{2} P_{j}}{d \tau_{j}^{2}}=\Sigma_{j^{\prime}=1}^{N} R_{j, j^{\prime}} P_{j^{\prime}}-T_{j},
$$

where $j$ and $j^{\prime}$ are discrete frequency-angle points. To apply that method of solution to the present problem, the specific forms of $R_{j, j^{\prime}}$ and $T_{j}$ must be given, as well as the explicit boundary conditions. In the present application (cf. Equations 2.2 and 2.3),

$$
R_{j, j^{\prime}}=1-\frac{2}{\kappa_{\nu}+\sigma_{\mathrm{T}, \mathrm{r}}} \frac{d \sigma_{\mathrm{T}, \mathrm{r}}}{d \Omega}\left(\mu_{j}, \mu_{j^{\prime}}\right) a_{j^{\prime}}
$$

and

$$
T_{j}=\frac{\kappa_{\nu}}{\kappa_{\nu}+\sigma_{\mathrm{T}, \mathrm{r}}} B_{\nu}(T(y)),
$$

where $a_{j^{\prime}}$ is the Gauss-Legendre quadrature weight for the angle $\mu_{j^{\prime}}$. Using the phase function for dipole scattering (Hummer 1962), the differential amount of scattering from directions $\mu^{\prime}$ into directions $\mu$ can be expressed as

$$
\frac{d \sigma_{\mathrm{T}, \mathrm{r}}}{d \mu^{\prime}}\left(\mu_{j}, \mu_{j^{\prime}}\right)=\sigma_{\mathrm{T}, \mathrm{r}} \frac{3}{16}\left(3+3 \mu_{j}^{2} \mu_{j^{\prime}}^{2}-\mu_{j}^{2}-\mu_{j^{\prime}}^{2}\right) .
$$

The boundary condition at the surface is simply $I_{-}=0$, i.e. no incident specific intensity. For the bottom boundary condition, the second order accurate expression $P_{\nu}=B_{\nu}$ is used, which is an approximation to the third order accurate 
diffusion approximation boundary condition suggested in the previous work (Auer 1976). The reason for choosing this specific bottom boundary condition is that it ensures that the specific intensity is isotropic at the bottom depth considered at each frequency, which will be useful when computing the Eddington factors in the temperature correction scheme.

Having specified $R_{j, j^{\prime}}, T_{j}$, and the boundary conditions, the radiative transfer problem can be solved using the method described in previous work (Auer 1976), giving $P_{\nu}(y, \mu)$ on the grid of depth and angle points used for each specific frequency. The emergent specific intensity at the surface is then $I_{\nu}(0, \mu)=$ $2 P_{\nu}(0, \mu)$

Under the isotropic scattering assumption the phase function is $g=1 / 2$ (Hummer 1962), and the expression for the differential amount of scattering becomes

$$
\frac{d \sigma_{\mathrm{T}, \mathrm{r}}}{d \mu^{\prime}}\left(\mu_{j}, \mu_{j^{\prime}}\right)=\sigma_{\mathrm{T}, \mathrm{r}} \frac{1}{2}
$$

The anisotropic expression is used throughout the present work, except in Section 4.2 , where the spectra computed with the anisotropic scattering expression are compared to those computed with the isotropic scattering expression.

\subsection{Temperature Correction}

The temperature correction scheme used is the same as that employed in a previous work (Z96), though a different discretization method is used. Both the temperature correction procedure and discretization method are described in full

in Appendix C. The temperature correction procedure requires the solution of the 
equation

$$
\frac{1}{k_{\nu}} \frac{d}{d y}\left(\frac{1}{k_{\nu}} \frac{d}{d y}\left(f_{\nu} J_{\nu}\right)\right)=\left(J_{\nu}-B_{\nu}\left(T_{0}\right)-\frac{d B_{\nu}}{d T} \frac{\int_{0}^{\infty}\left(J_{\nu}-B_{\nu}\left(T_{0}\right)\right) \kappa_{\nu} d \nu}{\int_{0}^{\infty} \frac{d B_{\nu}}{d T} \kappa_{\nu} d \nu}\right) \frac{1}{k_{\nu}},
$$

where

$$
f_{\nu}=J_{\nu}^{-1} \int_{0}^{1} \mu^{2} P_{\nu}(y, \mu) d \mu
$$

is one of the Eddington factors, and

$$
J_{\nu}=\int_{0}^{1} P_{\nu}(y, \mu) d \mu
$$

is the mean specific intensity. Note, however, that this is the mean specific intensity subject to the constraint of constant energy flux through the atmosphere, which will only equal the mean specific intensity from the radiative transfer calculation in the limit of zero temperature correction. The boundary condition at the surface is

$$
\frac{1}{k_{\nu}} \frac{d}{d y}\left(f_{\nu} J_{\nu}\right)=h_{\nu} J_{\nu}
$$

where

$$
h_{\nu}=J_{\nu}^{-1} \int_{0}^{1} \mu P_{\nu}(y, \mu) d \mu
$$

is another Eddington factor, and the boundary condition at the largest column depth is $J_{\nu}=B_{\nu}$.

Note that allowing for anisotropic scattering in the present work does not change form of equation 3.8, since the angular integral over the source function must come out to the same whether or not it has an angular dependence (all radiation 'absorbed' due to scattering opacity must be 're-emitted'). 
The temperature correction $\Delta T(y)$ is equal to the ratio of the two integrals in the last term of Equation 3.8 (see Equation C.10). To find $J_{\nu}$, and thus $\Delta T$, Equation 3.8 is solved using the $f_{\nu}$ computed in the radiative transfer problem. For the depth points beyond the maximum column depth considered at each frequency in the radiative transfer problem, the isotropic limit $f_{\nu}=1 / 3$ is used. A continuous $f_{\nu}$ is ensured by the use of the boundary condition $P_{\nu}=B_{\nu}$ in the radiative transfer problem.

Using a fixed $f_{\nu}(y)$, Equation 3.8 can be solved using a Rybicki type solution (Rybicki 1971), the details of which are given in Appendix C. The resulting temperature correction is applied to the temperature profile, and the radiative transfer repeated to give an updated $f_{\nu}(y)$, which in turn is used to correct the temperature profile. Iteration is continued until the maximum computed fractional temperature change is less than a specified threshold $\left(10^{-4}\right.$ throughout the present work). 


\section{CHAPTER 4 RESULTS}

This section presents results from running the first version (v1) of the McPHAC code, which is described in the present work. The aim is to present the capabilities and limitations of the present version of the code, to inspire future improvements and applications. Thus, previous works (Z96; G02; H06) are referred to for a more complete discussion of the hydrogen atmosphere structure and emergent spectrum.

The code has been compiled and run on an AMD platform running the Linux operating system. For details on the code implementation and numerical packages used, see Appendix D.

Unless otherwise stated, the following 'default' level of discretization is used: the number of frequency points used is $K=100$, the number of angle points used is $M=40$, the number of depth points used in the radiative transfer calculation is $N_{\text {rad }}=3200$, and the number of depth points used in the temperature correction procedure is $N_{\text {temp }}=3200$. Further, the minimum column depth considered at $T_{\text {eff }}=10^{6.5} \mathrm{~K}$ is $y_{\text {min }}=10^{-5} \mathrm{~g} / \mathrm{cm}^{2}$, while it is $y_{\min }=10^{-9} \mathrm{~g} / \mathrm{cm}^{2}$ at all lower effective temperatures. For all effective temperatures the maximum column depth

is set to $y_{\max }=10^{2} \mathrm{~g} / \mathrm{cm}^{2}$, though this may be increased by the code as described in Section 3.1 . 
At the 'default' discretization, the time required for one iteration (radiative transfer plus temperature correction) is approximately half an hour, giving a total running time for McPHAC of 2-3 hours. The radiative transfer calculation is expected to scale roughly as $K N_{\text {rad }} M^{3}$, while the temperature correction is

expected to scale roughly as $K N_{\text {temp }}{ }^{2}+N_{\text {temp }}{ }^{3}$. These scalings have not been verified in a systematic way, but the time required to run McPHAC for different discretization levels did appear to follow similar scalings.

\subsection{Emergent Spectra}

Normalized and rescaled spectra computed for various effective temperatures are shown in Figure 4-1, along with a blackbody (BB) spectrum for comparison. The default discretization level described previously is used, with the exception of the $T_{\text {eff }}=10^{6.5} \mathrm{~K}$ spectrum, for which $M=160$ angle points were used. The hottest of the computed spectra lies the closest to the BB spectrum, and the spectra get harder with decreasing effective temperature. Thomson scattering is expected to be more important in the hotter atmospheres, and the relative magnitudes of the free-free and reduced Thomson opacities as a function of frequency appears to be important in determining the shape of the spectrum. The spectra are significantly harder than a blackbody spectrum because of the frequency dependence of the free-free opacity (the free-free cross-section decreases with increasing frequency), which means that the flux at a higher frequency originates deeper in the atmosphere, where the temperature is greater. For higher effective temperatures, Thomson scattering is more important since the spectrum is shifted to higher energies, thus reducing the free-free opacity. Since the reduced 
Thomson opacity is frequency independent, it softens the spectrum by limiting the depth in the atmosphere from which the high-energy side of the spectrum originates. The behavior described here is similar to that seen in previous work (H06), where the high-energy side of the rescaled spectrum steepened with increasing effective temperature.

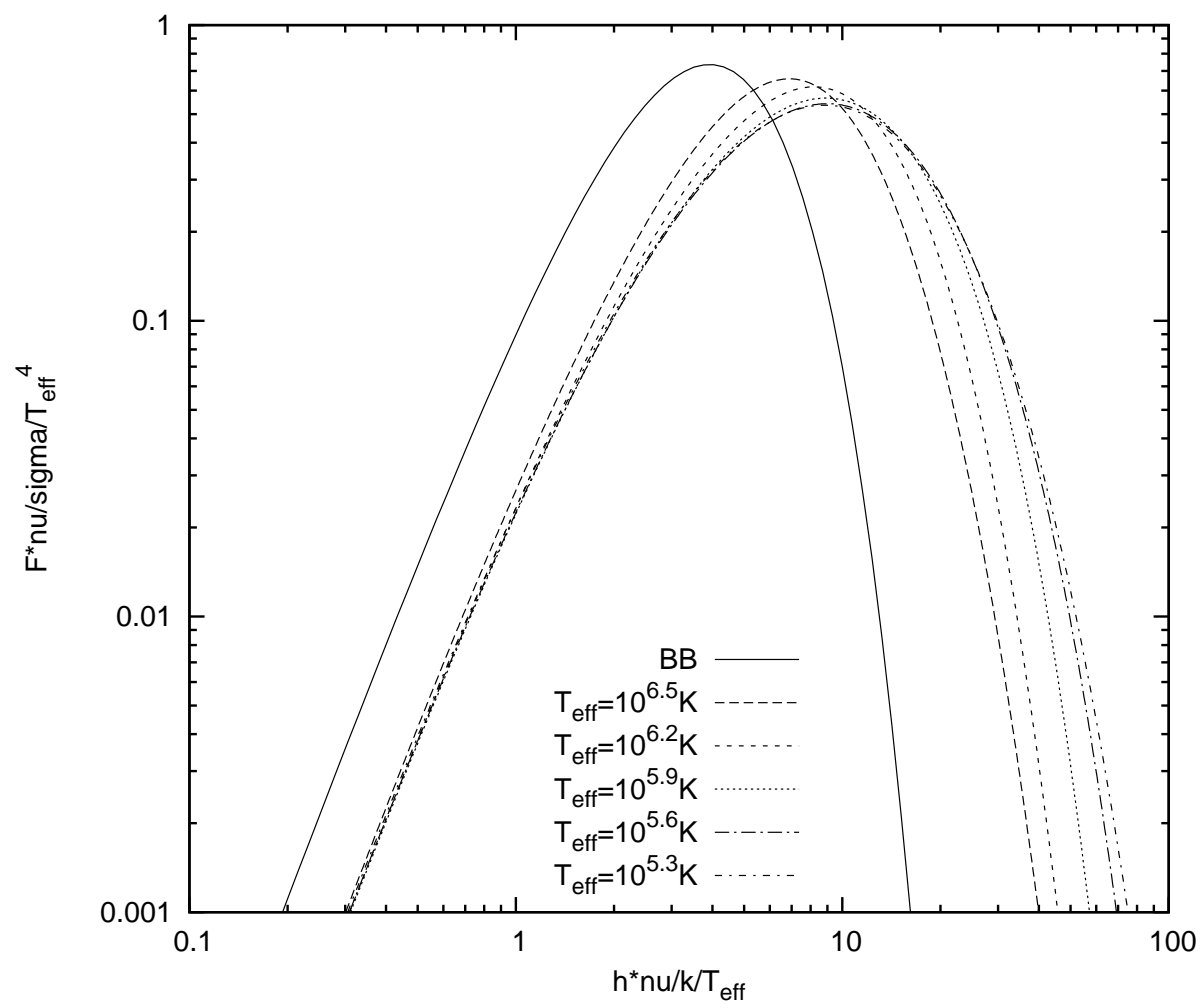

Figure 4-1: Normalized spectra computed for various effective temperatures, along with a blackbody (BB) spectrum for comparison. The hottest of the computed spectra is the closest to the blackbody spectrum, and the spectra get harder with decreasing effective temperature.

\subsection{Anisotropic Thomson Scattering}

The present work allows for Thomson scattering to be treated anisotropically, in contrast to previous work (Z96; G02; H06). The effect of relaxing the isotropy 
assumption is investigated here, by comparing the spectra computed with the McPHAC code using the anisotropic scattering expression to those computed using the isotropic scattering expression (cf.Section 3.2).

Figure 4-2 shows the ratio of the spectrum calculated considering anisotropic Thomson scattering to that computed assuming isotropic scattering, for various effective temperatures. Considering anisotropic scattering appears to increase the flux above $1 \mathrm{keV}$, with the largest increase happening around $5 \mathrm{keV}$ (apparently insensitive to effective temperature). The effective temperatures of each pair of spectra differ by less than $2 \times 10^{-5}$. Thus, increasing the flux above $1 \mathrm{keV}$ reduces the flux below $1 \mathrm{keV}$ to keep the effective temperature the same. Corrections to anisotropic Thomson scattering due to collective effects (Boercker 1987) may change the importance of considering anisotropic scattering, but it appears that isotropic scattering is an adequate assumption as long as one is not seeking accuracy better than $1 \%$ in flux as a function of photon energy.

\subsection{Convergence of Computational Method}

There are multiple sources of uncertainty in the spectra computed under the assumptions described in Chapter 2: uncertainty in the input parameters (Gaunt factor, physical constants, etc.), truncation (discretization) uncertainty in the numerical method (both for the temperature correction and radiative transfer), and the convergence uncertainty between iterations. Investigation of the effect of uncertainty in the input parameters is left for future work, as the aim of the present work is limited to presenting the computational capabilities of the McPHAC code, while the latter two forms of uncertainty are investigated here. 


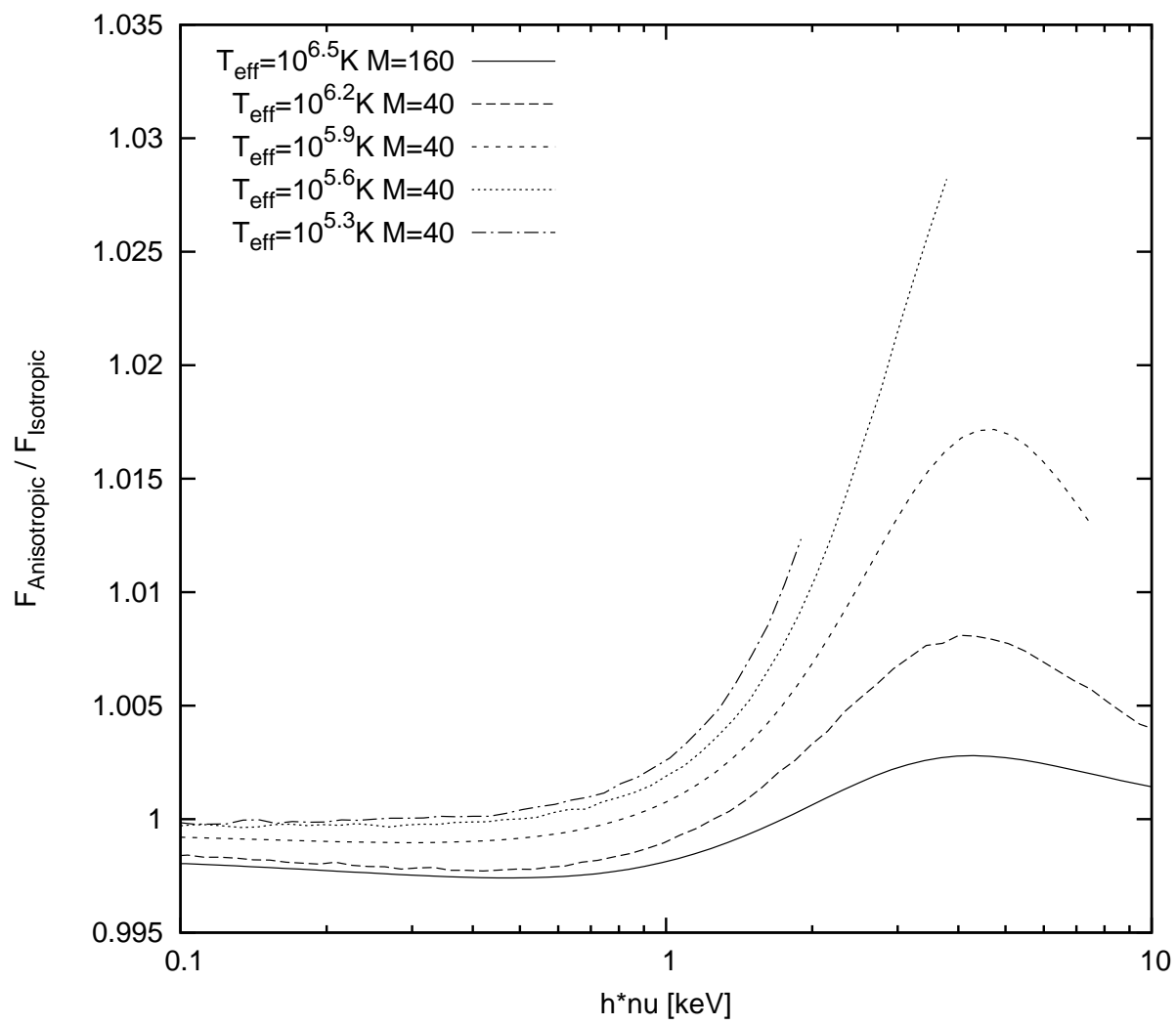

Figure 4-2: Ratio of the spectrum calculated considering anisotropic Thomson scattering to that computed assuming isotropic scattering, for various effective temperatures. The curves terminate at $h \nu=120 k T_{\text {eff }}$, which corresponds to the highest frequency considered. Considering anisotropic scattering appears to increase the flux above $1 \mathrm{keV}$, with the largest increase happening around $5 \mathrm{keV}$ (apparently insensitive to effective temperature). The effective temperatures of each pair of spectra differ by less than $2 \times 10^{-5}$. Thus, increasing the flux above 1 $\mathrm{keV}$ reduces the flux below $1 \mathrm{keV}$ to keep the effective temperature the same.

The truncation error is the error introduced by truncating the power series describing a function to a finite order. The local truncation error from one point to another can usually be constrained, but the additive effects of truncation errors are more difficult to quantify. One way to estimate the size of the truncation error is to perform the calculation for an increasingly fine discretization, and to analyze 
the convergence of the resulting value of interest. In general, the truncation error in each equation due to the discretization of each variable should be investigated, so a multi-dimensional grid of discretization levels should be used for each of the input parameters for which spectra are computed. However, at present a more limited approach is taken, intended mainly to give a sense of the capabilities and limitations of the code. A more thorough treatment should be performed in specific applications where it is important to constrain the uncertainty in the computed spectra.

The discretization of column/optical depth in the radiative transfer calculation is perhaps the most obvious example of how a truncation error can be introduced in the computed spectrum. To constrain this, the radiative transfer calculation was repeated for a fixed atmospheric structure, varying $N_{\text {rad }}$ while keeping all other discretizations at their default values. Figure 4-3 shows the convergence of the computed specific intensity at the surface with increasing $N_{\text {rad }}$, for a frequency close to the peak of the $T_{\text {eff }}=10^{6.5} \mathrm{~K}$ spectrum. The specific intensities shown are those computed after six iterations, where the maximum fractional temperature change in the atmosphere in the last iteration is less than $10^{-4}$. As the number of depth points in the radiative transfer calculation is increased from 100 to 1600 , the fractional difference in the computed spectra falls below $10^{-4}$, indicating that the truncation (discretization) error in the radiative transfer calculation due to discretizing the depth variable is smaller than $10^{-4}$ when using 3200 depth points. Hardly any dependence on $\mu$ is seen in the convergence, indicating that convergence can be demonstrated separately for the discretizations in $\mu$ and 
in depth, rather than resorting to a multidimensional grid of discretization levels. Further, this indicates that a single component may limit the convergence at every angle. Identifying this component and finding some way to reduce it could improve convergence. However, even limited by this hypothetical component, second-order convergence is observed.

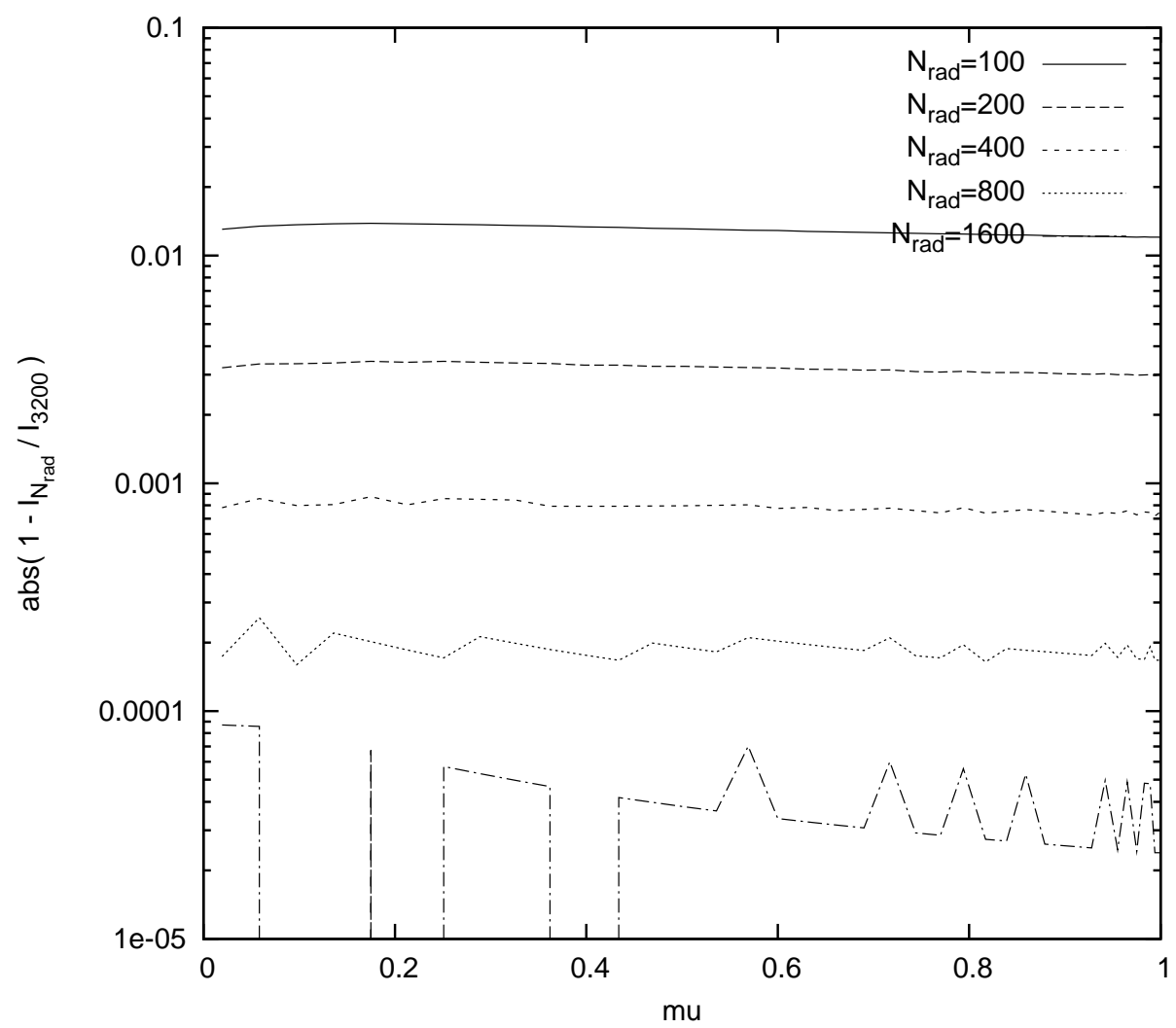

Figure 4-3: Convergence of the specific intensity resulting from repeating the radiative transfer calculation for an increasing number of depth points. The effective temperature of the spectrum is $T_{\text {eff }}=10^{6.5} \mathrm{~K}$, and the specific intensity shown is for a frequency close to the spectrum peak $(k=3 K / 5)$. Note that the convergence scales approximately as $1 / N_{\text {rad }}^{2}$, and that doubling $N_{\text {rad }}$ thus decreases by a factor of four the fractional difference between the specific intensity computed for $N_{\text {rad }}$ depth points and that computed using 3200 depth points. 
Figure 4-4 is the same as Figure 4-3, but for the highest frequency considered, for which the largest fractional difference is seen between the spectra computed with 1600 and 3200 depth points. Even at this frequency, the truncation (discretization) uncertainty in the spectrum due to the discretization of the depth variable extrapolates to below $10^{-4}$ when using $N_{\text {rad }}=3200$. Again, second order convergence is seen.

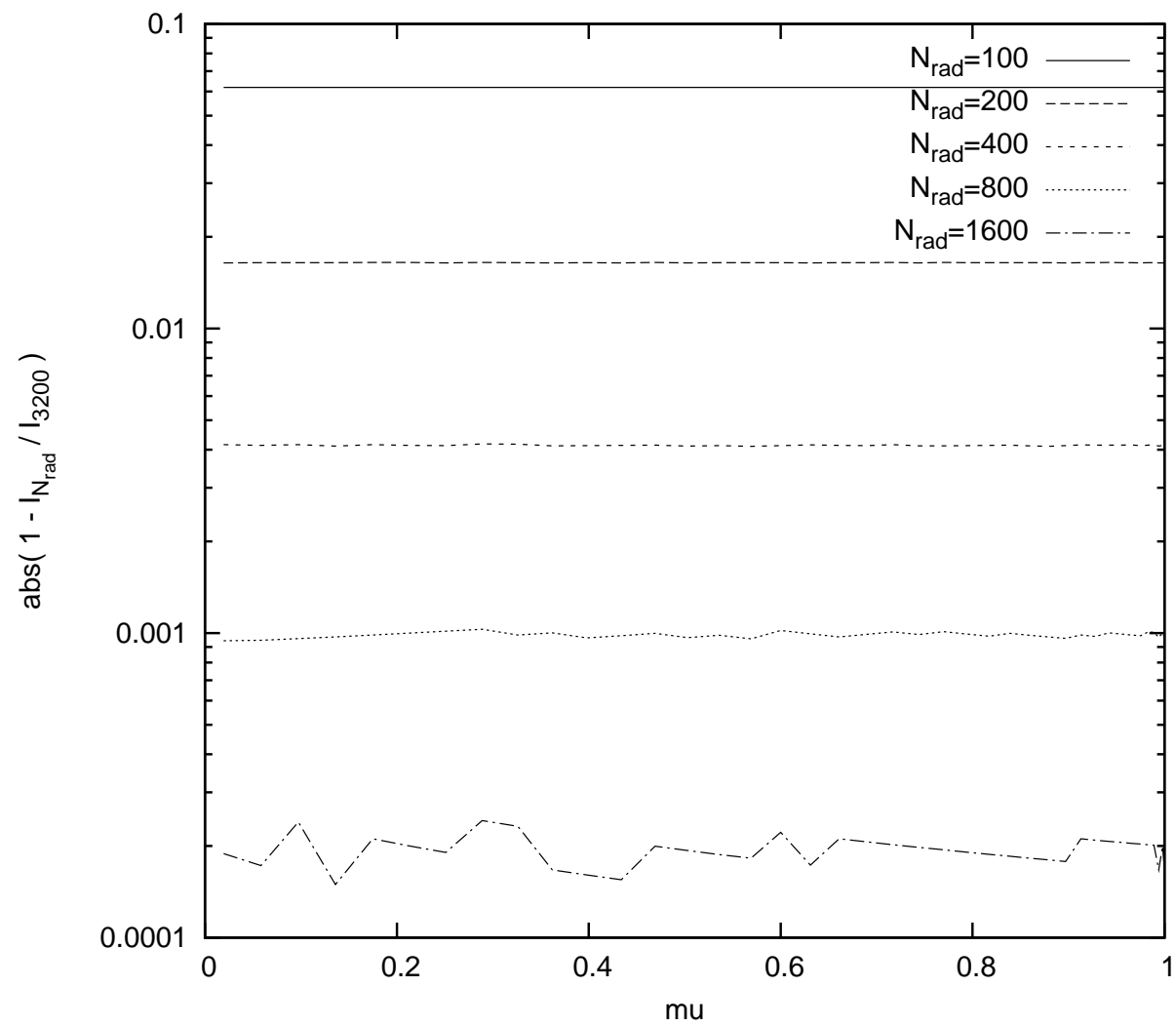

Figure 4-4: Same as Fig. 4-3, but for the highest frequency considered $(k=K)$. This is the frequency for which the largest fractional difference is seen between the spectra computed with 1600 and 3200 depth points. Note that as in Figure 4-3, the convergence scales approximately as $1 / N_{\text {rad }}^{2}$. 
Another truncation error that may be important in the radiative transfer calculation is that introduced through the discretization of the angle variable $\mu$. To constrain just the truncation error in the radiative transfer calculation due to discretizing $\mu$, the radiative transfer calculation would be repeated for a fixed atmosphere structure as is done for the number of depth points. However, at present, code to do this has not been implemented, so in stead the entire iterative solution is repeated for an increasing number of angle points $M$. The convergence of the computed spectrum with number of angle points is shown in Figure $4-5$, for $T_{\text {eff }}=10^{6.5} \mathrm{~K}$. The spectra shown are those computed after six iterations, where the maximum fractional temperature change in the atmosphere in the last iteration is less than $10^{-4}$. From the rate of convergence seen, it is expected that the uncertainty in the spectrum due to discretizing the $\mu$ variable is less than $10^{-4}$ when using 160 angle points (for this effective temperature). When varying the number of angle points, the entire calculation was run for each number of angle points. This is in contrast to the procedure used when varying the number of depth points, where only a single part of the calculation (temperature correction or radiative transfer calculation at a specific iteration) was repeated for different numbers of depth points. Thus, the convergence uncertainty investigated using this figure includes not only truncation uncertainty in the computed spectrum introduced by discretizing $\mu$, but also the effect of uncertainty in the computed Eddington factors on the temperature correction between iterations. The individual truncation uncertainties on the Eddington factor due to 
discretizing $y$ and $\mu$ have not been investigated, so this should be done in future work.

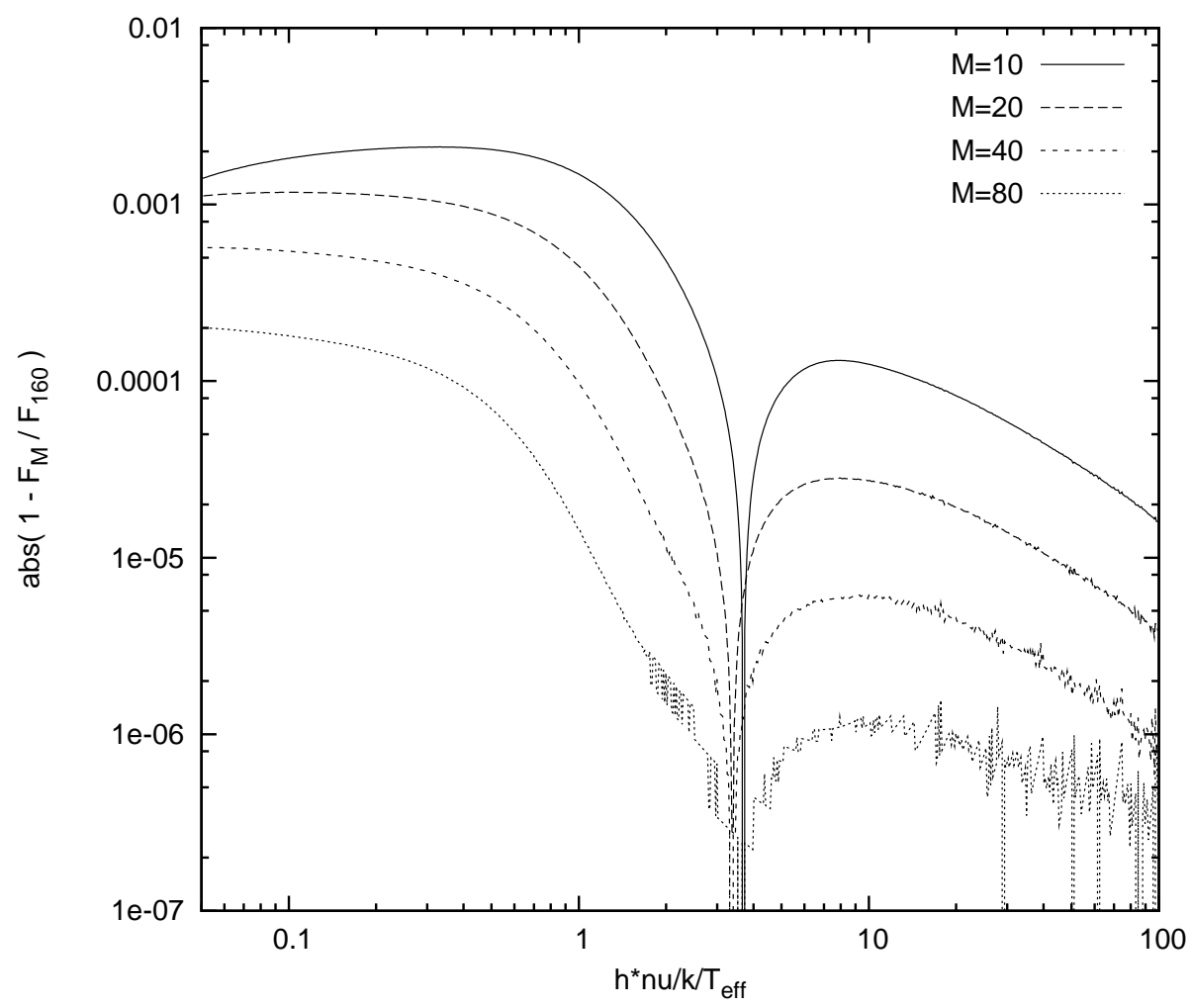

Figure 4-5: Convergence of the emergent spectrum resulting from re-running the full McPHAC code for an increasing number of angle points. The effective temperature of the spectrum is $T_{\text {eff }}=10^{6.5} \mathrm{~K}$. There appears to be a sign change of $1-F_{\mathrm{M}} / F_{160}$ near $h \nu / k / T_{\text {eff }} \sim 3.5$ for all values of $M$, which seems to indicate that the discretization of $\mu$ leads to consistent over- or under-prediction of the flux on either side of $h \nu / k / T_{\text {eff }} \sim 3.5$. It is not clear why the discretization leads to this consistent this over- or under-prediction, but this could be investigated in future work.

In calculating the temperature correction at each iteration, the truncation error due to discretizing the depth variable may be important. To investigate this, the temperature correction at each iteration is repeated for a varying number 
of depth points. The convergence of the temperature profile (after temperature correction) computed with $N_{\text {temp }}$ depth points is shown in Figure 4-6, for a $T_{\text {eff }}=10^{6.5} \mathrm{~K}$ atmosphere. The first iteration is shown, since this is where the largest temperature correction is computed, but the corresponding figures for later iterations look almost identical. For anything more than 800 depth points, the truncation uncertainty in the temperature correction procedure introduced by discretizing $y$ is less than $10^{-4}$ (at this effective temperature).

Separate from the truncation uncertainty, there is the convergence uncertainty. The convergence uncertainty is the uncertainty introduced by solving coupled equations iteratively, since the solution at a given iteration is different from that that would be obtained in the limit of an infinite number of iterations. The convergence uncertainty can be constrained by analyzing the convergence of the resulting values for successive iterations.

The temperature profile is corrected at each iteration, and these corrections affect the structure of the atmosphere and the emergent spectrum. Thus, it is important that enough iterations are used to bring the largest fractional temperature change below some threshold. The convergence of the computed fractional temperature change with iteration is shown in Figure $4-7$, for a $T_{\text {eff }}=10^{6.5} \mathrm{~K}$ atmosphere. At five iterations, the maximum computed fractional temperature change is below $10^{-4}$

It is not obvious what the overall effect on the computed spectrum is of a given fractional temperature change, so to constrain the total convergence uncertainty on the computed specific intensity at the surface, the convergence 


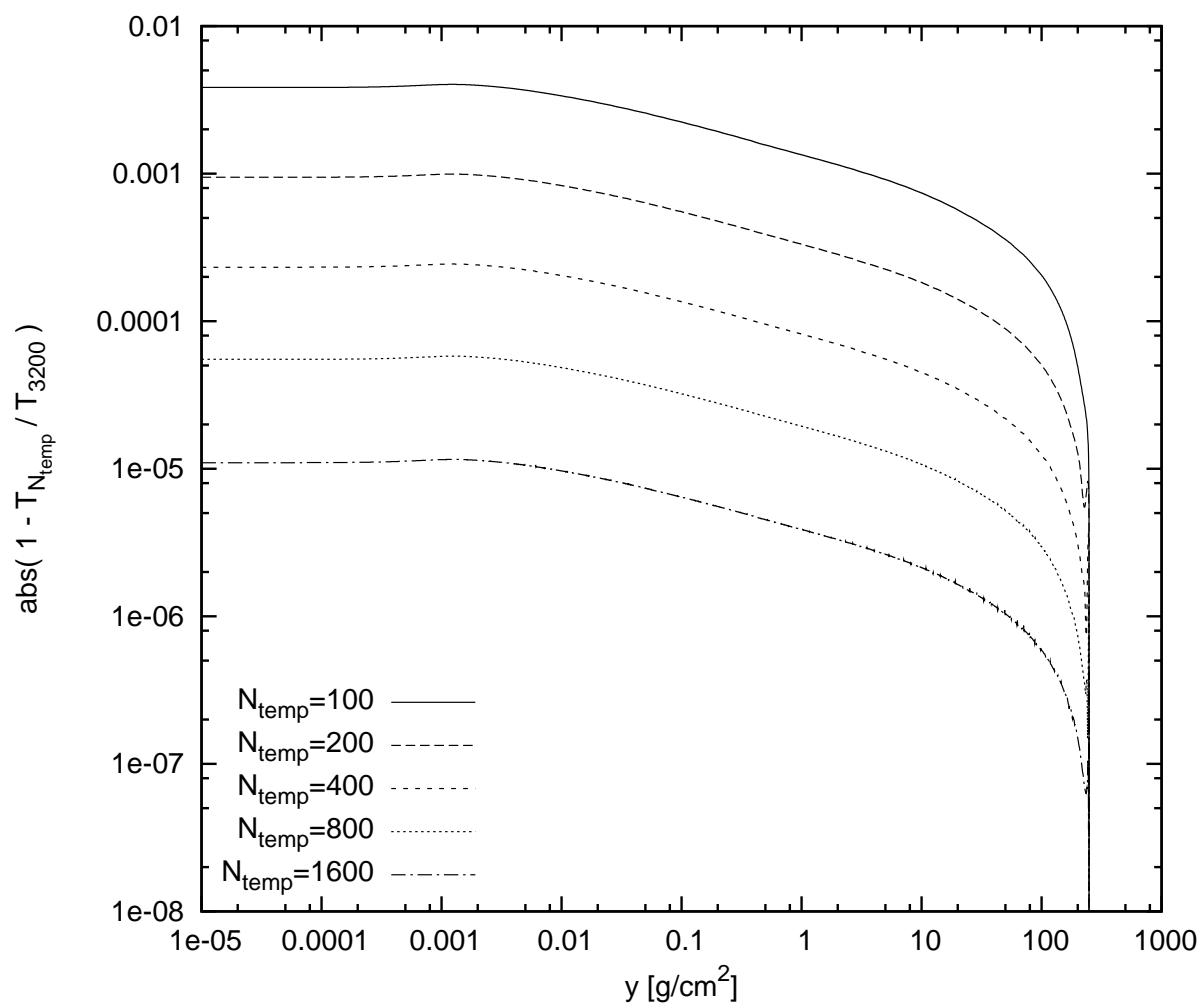

Figure 4-6: Convergence of the temperature profile resulting from repeating the temperature correction procedure with an increasing number of depth points. The flux through the atmosphere corresponds to $T_{\text {eff }}=10^{6.5} \mathrm{~K}$. The first iteration is shown, since this is where the largest temperature correction is computed, but the corresponding figures for later iterations look almost identical. Note that the convergence scales approximately as $1 / N_{\text {temp }}^{2}$, and that doubling $N_{\text {temp }}$ thus decreases by a factor of four the difference between the temperature profile computed with $N_{\text {temp }}$ depth point and that computed with 3200 depth points. Note that the sharp drop-off around $y=300 \mathrm{gcm}^{-2}$ is due to the bottom boundary condition, which results in zero computed temperature correction there.

of the computed specific intensity with iteration should be investigated. This convergence is shown in Figure 4-8, for a $T_{\text {eff }}=10^{6.5} \mathrm{~K}$ atmosphere. The changes seen are due to structure changes in the atmosphere between iterations, and show 


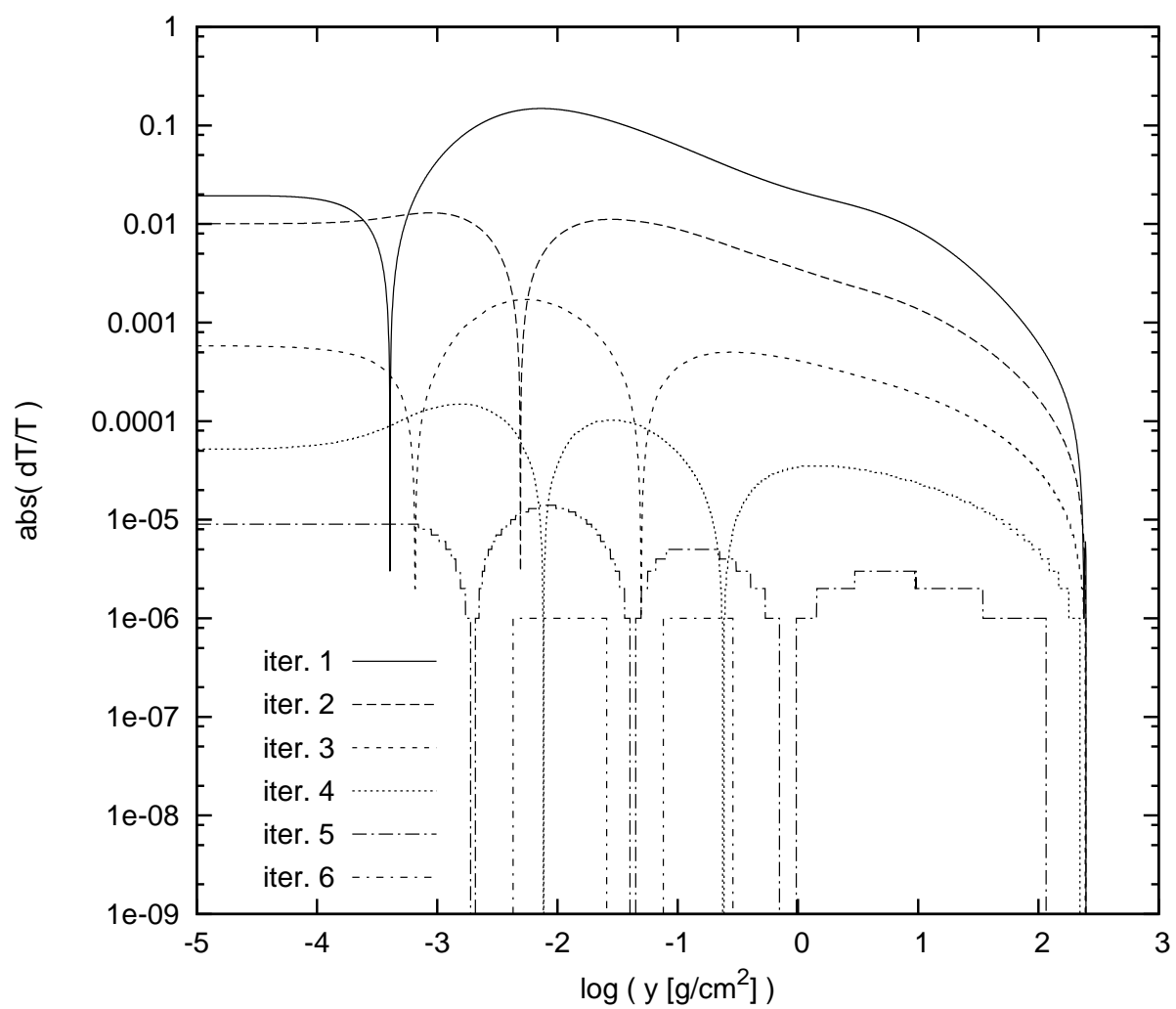

Figure 4-7: The computed fractional temperature change ( $\Delta T$, cf. Equation C.10) with depth, for each iteration. The flux through the atmosphere corresponds to $T_{\text {eff }}=10^{6.5} K$. The sharp decrements (e.g., near $\log (y)=-3.5$ for iteration 1 ) are due to a change in sign of $\Delta T$.

that the uncertainty in the specific intensity at the surface due to the iteration scheme used is less $10^{-4}$ after five iterations (for this effective temperature).

The most intuitive way to calculate the outward flux at the surface (the emergent spectrum), is the integral $F_{\nu}=\int_{0}^{1} \mu I_{\nu}(\mu) d \mu$. However, it can also be computed from the Eddington factor $f_{\nu}$ and mean specific intensity $J_{\nu}$ through the expression (Z96; cf. Appendix C)

$$
F_{\nu}=\frac{4 \pi}{\alpha_{\nu}+\sigma_{\nu}} \frac{d}{d y}\left(f_{\nu} J_{\nu}\right)
$$




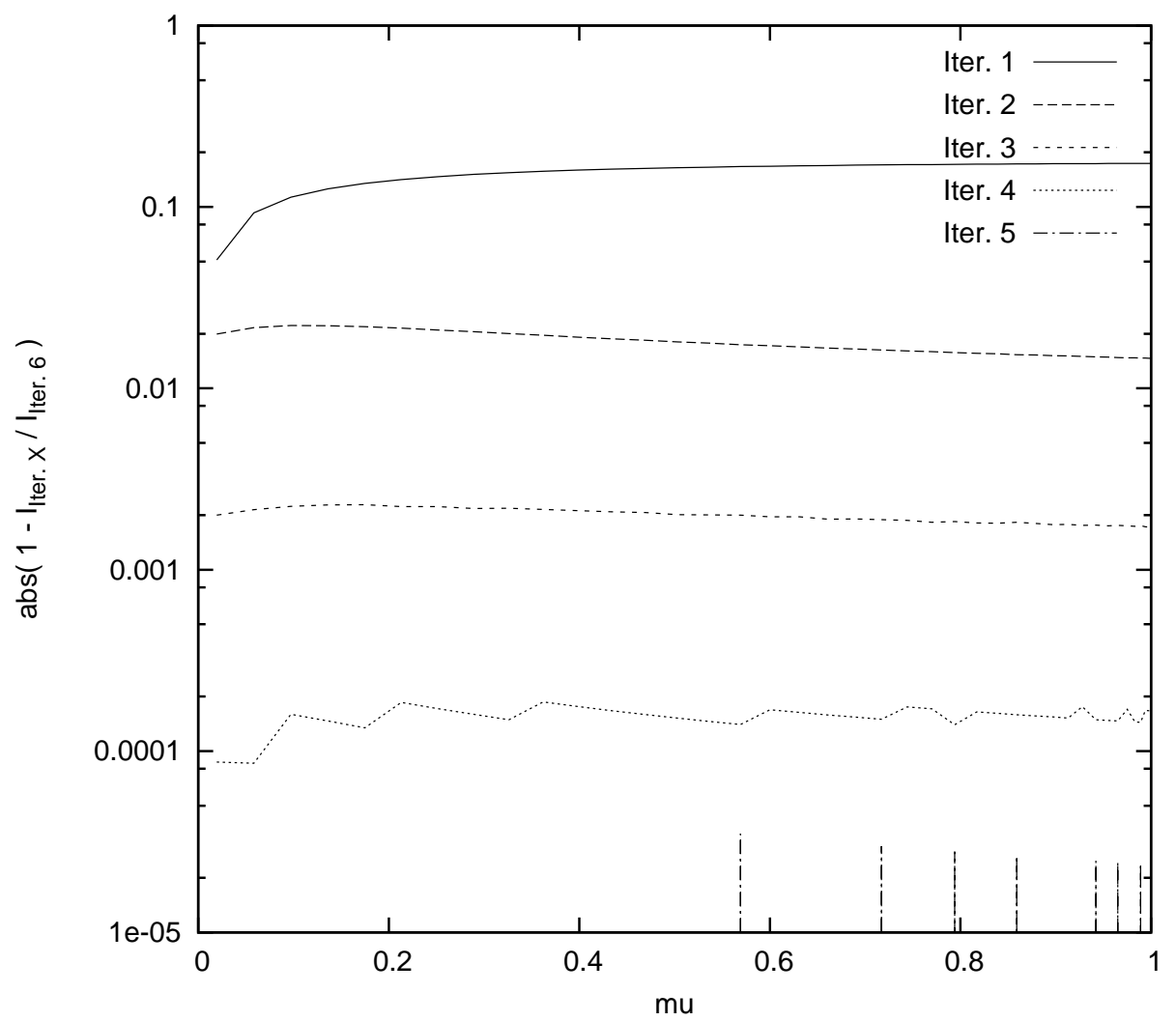

Figure 4-8: Convergence of the specific intensity as the temperature profile is iteratively corrected. The effective temperature of the spectrum is $T_{\text {eff }}=10^{6.5} \mathrm{~K}$, and the specific intensity shown is for a frequency close to the spectrum peak $(k=3 K / 5)$.

Interestingly, $J_{\nu}$ is calculated in the temperature correction procedure, and in the limit of zero computed temperature correction, that $J_{\nu}$ is the same as that computed in the radiative transfer calculation. Thus, if the Eddington factors can be computed to sufficient accuracy in a computationally efficient way, it is possible that it is computationally advantageous to use the spectrum calculated from the temperature correction procedure, rather than require sufficient sufficient accuracy of the calculated spectrum in the radiative transfer calculation. Figure 
4-9 compares the normalized spectrum computed from the temperature correction procedure to that computed using in the radiative transfer calculation. The spectra shown are those computed after six iterations, where the maximum fractional temperature change in the atmosphere in the last iteration is less than $10^{-4}$. The number of angle points $M$ used for each curve is indicated in the legend. The two curves (40 and 160 angle points) for $T_{\text {eff }}=10^{6.5} \mathrm{~K}$ are almost identical, and show that the spectra computed using the two different methods differ by less than $10^{-4}$ for $h \nu /\left(k T_{\text {eff }}\right)<50$. This indicates that the difference between the spectra computed with the radiative transfer calculation and with the temperature correction procedure is not sensitive to the discretization number of angle points used. Further, the spectrum computed with the temperature correction procedure can be made accurate enough to be used in place of that computed in the radiative transfer calculation. The difference between the two spectra at the highest frequencies may be due to there being a significant contribution to the flux at the surface from greater than 80 optical depths at these frequencies, as this flux would be ignored in the radiative transfer calculation. The curve for $T_{\text {eff }}=10^{5.6} \mathrm{~K}$ shows a larger difference between the spectra computed with the two different methods, which may be due to the larger logarithmic range of column depths used at this effective temperature leading to a larger truncation error on the spectrum computed in the temperature correction procedure (since the number of depth points is fixed). 


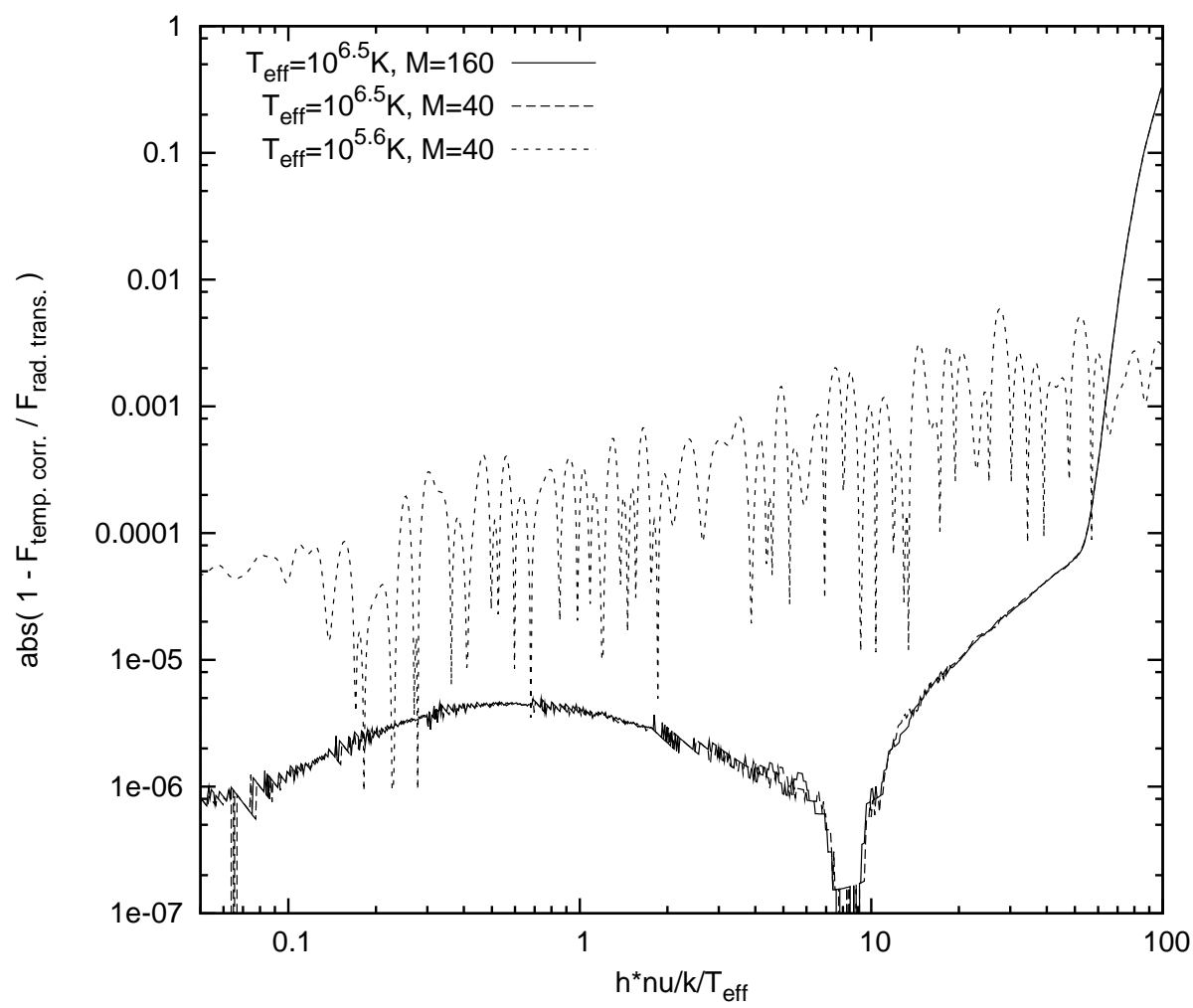

Figure 4-9: Comparison of the emergent spectrum resulting from the radiative transfer calculation to that resulting from the temperature correction procedure. The number of angle points used for each curve is indicated in the legend. The two curves (40 and 160 angle points) for $T_{\text {eff }}=10^{6.5} \mathrm{~K}$ are almost identical.

\subsection{Comparison with Previous Work}

Figure 4-10 shows a comparison of the normalized spectra computed using the McPHAC code to those of previous work (Z96), for various effective temperatures. There appears to be generally good agreement between the spectra, though the spectra computed in previous work predict significantly less flux at high energies than those of the present work for the three lowest effective temperatures. The additional difference seen near the peak of the two $T_{\text {eff }}=10^{5.3} \mathrm{~K}$ spectra is 
expected to be due to the assumption of complete ionization in McPHAC, as the presence of neutral hydrogen alters the shape of the Rayleigh-Jean side of the spectrum for this and lower effective temperatures (cf. Figure 5 in the previous work).

Figure 4-11 shows the ratio of the pairs of spectra shown in Figure 4-10. The spectra are interpolated to allow for comparison. All the curves show a sharp drop-off at the high-frequency end. At frequencies below this drop-off, all but the coolest spectra computed using the McPHAC code agree with those of previous work (Z96) at the two-percent level. Little flux is expected at the energies above the drop-off, so this difference is unlikely to have practical consequences for most applications. An exception is 1RXS J185635.1-375433: If the spectra computed in previous work predict too low flux in the X-ray band, this could affect conclusions based on fitting to that model.

One possible cause for the lower flux seen at the highest energies in the spectra of the previous work (Z96) is that the previous work considered a smaller maximum column depth than the present work. To investigate this hypothesis, the McPHAC code was run for the column depth ranges used in the previous work for three of the effective temperatures (read from a figure in the previous work). Figure 4-12 shows the normalized spectrum of the previous work, the McPHAC computed spectrum using the default column depth range ('large tau'), and the McPHAC computed spectrum using the the column depth range of the previous work ('Z96 tau'), for the three effective temperatures where a clear difference in the spectra was seen in Figure 4-10. The 'Z96 tau' McPHAC spectra agree well 


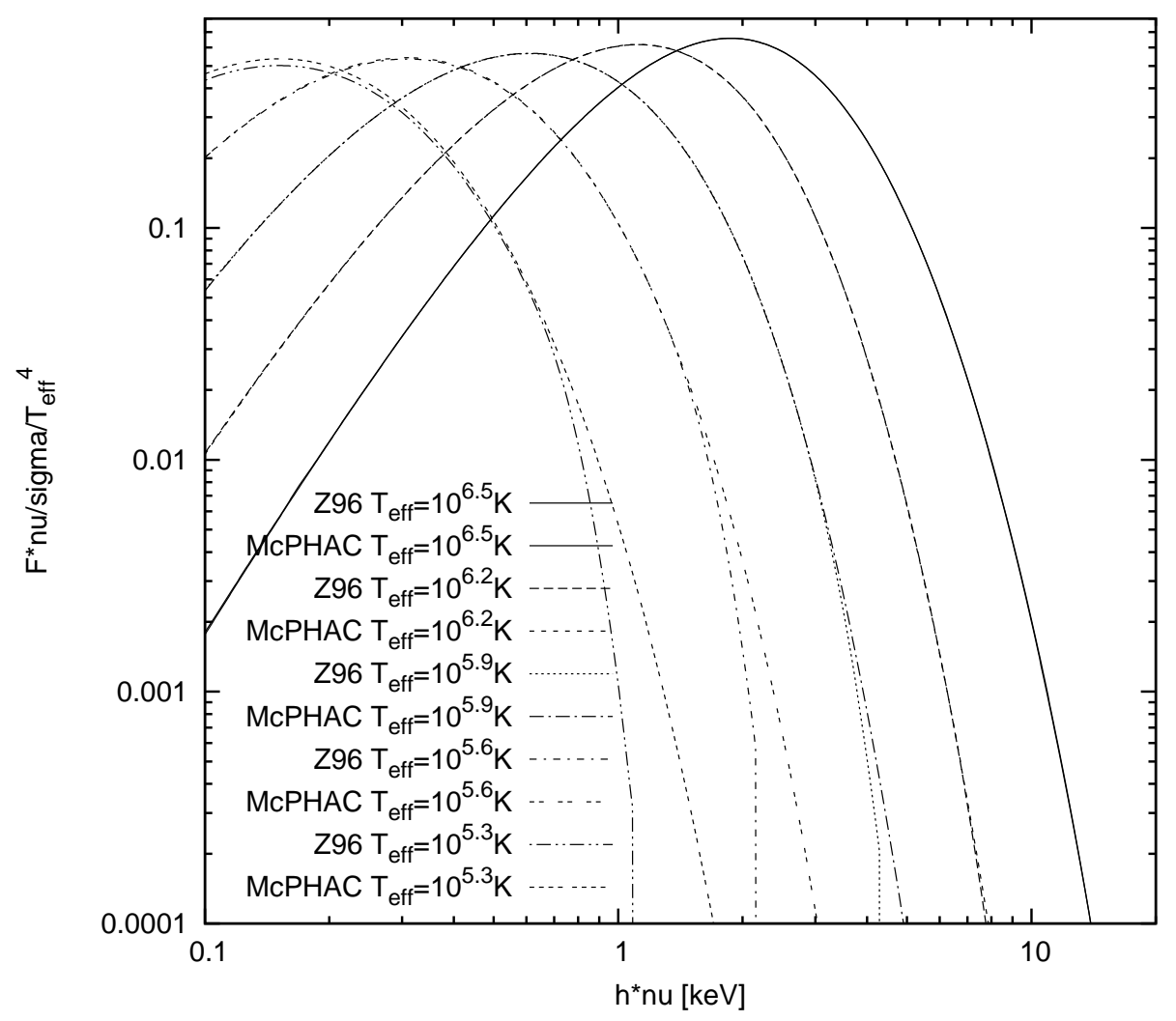

Figure 4-10: Comparison of the normalized spectra computed using McPHAC to those of previous work (Z96), for various effective temperatures. The vertical high-frequency cut-offs seen for the three lowest effective temperature spectra of the previous work are present because the tabulated spectra are not specified at frequencies above the cut-off value. There generally is good agreement between the McPHAC spectra and those of the previous work, though the McPHAC spectra predict significantly more flux at high energies than those of the previous work for the three lowest effective temperatures. The additional difference seen near the peak of the two $T_{\text {eff }}=10^{5.3} \mathrm{~K}$ spectra is expected to be due to the assumption of complete ionization in McPHAC, as the presence of neutral hydrogen alters the shape of the Rayleigh-Jean side of the spectrum for this and lower effective temperatures (cf. Figure 5 in the previous work).

with those of the previous work, indicating that the difference between these and the 'large tau' McPHAC spectra is due to the previous work considering too few 


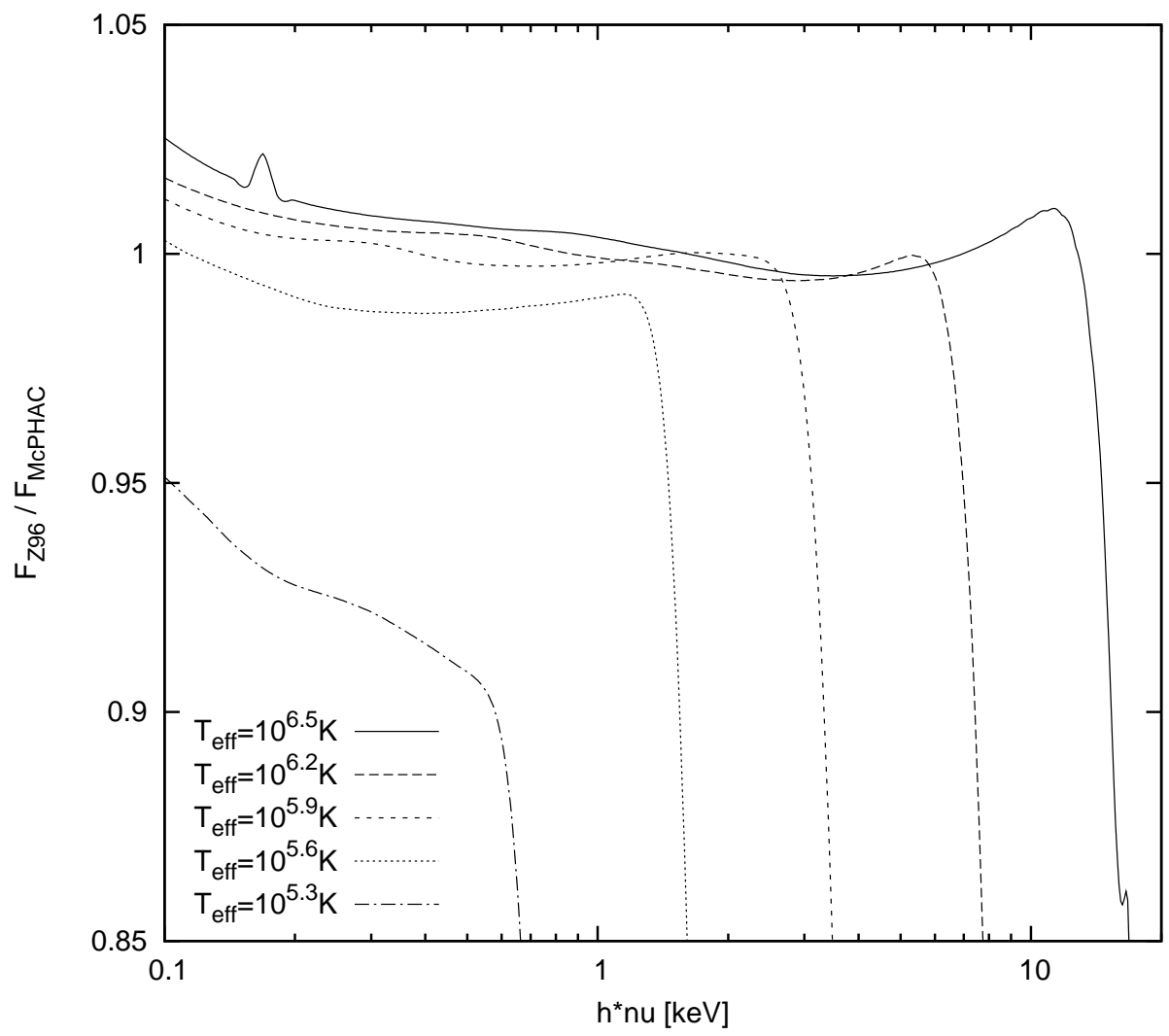

Figure 4-11: Ratio of the pairs of spectra shown in Fig. 4-10. All the curves show a sharp drop-off at the high-frequency end. At frequencies below this drop-off, all but the coolest spectra computed using the McPHAC code agree with those of previous work (Z96) at the two-percent level.

optical depths at the highest frequencies. A fractional comparison between the 'Z96 tau' spectra and those of previous work would not be illuminating since the any differences in the high-frequency part of the spectrum could be attributed to not using the exact same column depth ranges, and any differences in the low energy part of the spectrum is already shown in Fig. 4-11. 


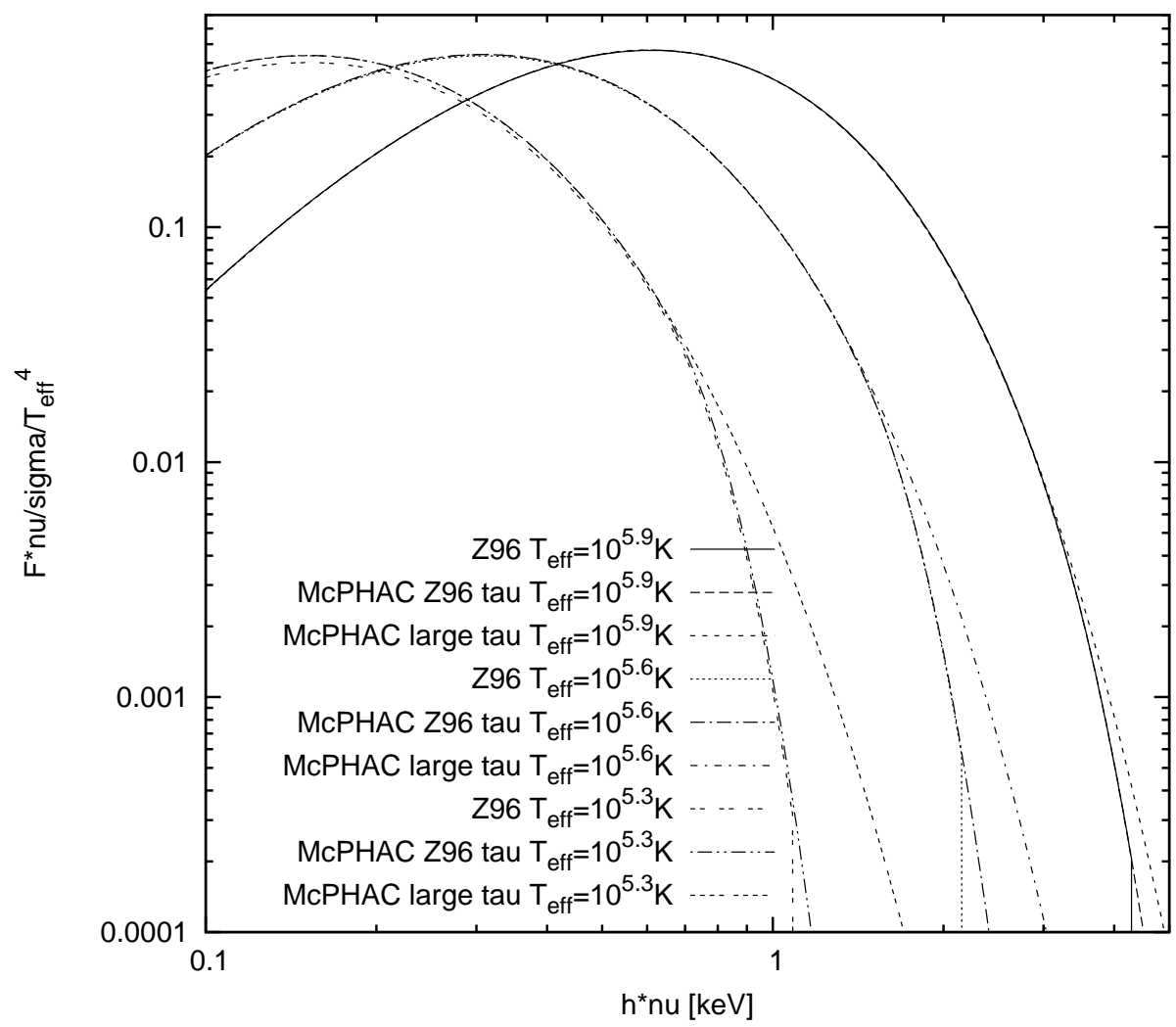

Figure 4-12: Comparison of the normalized spectra computed using McPHAC code to those of previous work (Z96), for various effective temperatures. The column depth range used for the McPHAC spectra labeled 'Z96 tau' at each effective temperature is taken to be the same as that used in the previous work. The column depth range for the McPHAC spectra labeled 'large tau' using 80 optical depths at all frequencies. The 'Z96 tau' McPHAC spectra agree well with those of the previous work, indicating that the difference between these and the 'large tau' $\mathrm{McPHAC}$ spectra is due to the previous work considering too few optical depths at the highest frequencies. A fractional comparison between the 'Z96 tau' spectra and those of previous work would not be illuminating since the any differences in the high-frequency part of the spectrum could be attributed to not using the exact same column depth ranges, and any differences in the low energy part of the spectrum is already shown in Fig. 4-11.

\subsection{Eddington Factors}

The Eddington factor $f_{\nu}\left(\tau_{\nu}\right)$, computed for a $T_{\text {eff }}=10^{6.5} \mathrm{~K}$ atmosphere using $M=160$ angle points, is shown in Figure 4-13. For isotropic specific intensity, 
the Eddington factor takes the value $f=1 / 3$. If the specific intensity is larger for small $\mu$ than for large $\mu$, then $f<1 / 3$. Conversely, if the specific intensity is larger for large $\mu$ than for small $\mu$, then $f>1 / 3$. As expected, $f$ goes to one third at the largest optical depth, where the radiation is expected to be practically isotropic. The largest deviation from $f=1 / 3$ occurs near the surface, for the peak frequency of the spectrum. For frequencies above the peak $f \gtrsim 1 / 3$, while for frequencies below the peak there are optical depths at which $f<1 / 3$. A possible explanation for this behavior is as follows: The specific intensity for small $\mu$ is set mainly by the blackbody function for the temperature at that depth. Since the temperature increases monotonically with increasing $\tau$, as does the the specific intensity for small $\mu$. The ingoing specific intensity for large magnitude $\mu$ depends on the amount of emitting material above the depth considered, and on the temperature and opacity of that material. Thus, the ingoing specific intensity for large magnitude $\mu$ should increase more slowly with optical depth than the specific intensity for small $\mu$. The outgoing specific intensity for large magnitude $\mu$ should not change by much in the top fraction of an optical depth of the atmosphere, so the Eddington factor should thus initially decrease with increasing optical depth from the surface. The magnitude of the outgoing specific intensity at large magnitude $\mu$ compared to the specific intensity at small $\mu$ determines whether there is a region where $f<1 / 3$ for a given frequency. For frequencies above the spectrum peak, the outgoing specific intensity for large magnitude $\mu$ appears to be large enough to keep $f \gtrsim 1 / 3$, while this is not the case for frequencies below the peak. The graph for the Eddington factor $h$ looks similar, except $h$ is $1 / 2$ for 
isotropic specific intensity, not $1 / 3$. Only the value of $h$ at the surface is used, however, so the depth dependence is not really of interest.

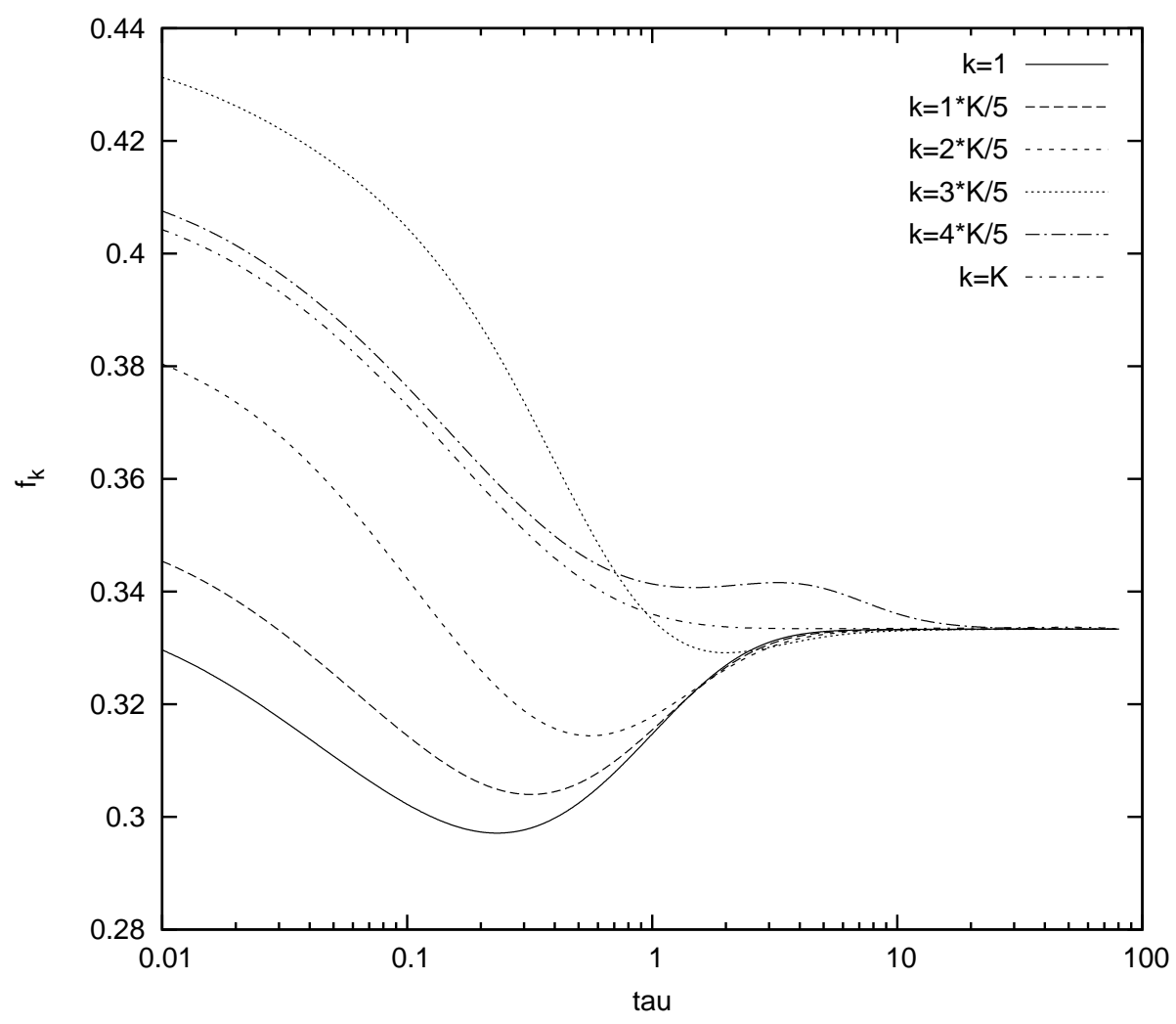

Figure 4-13: Eddington factor $f$, computed for a $T_{\text {eff }}=10^{6.5} \mathrm{~K}$ atmosphere. The largest deviation from $f=1 / 3$ occurs near the surface, for the peak frequency of the spectrum. For frequencies above the peak $f \gtrsim 1 / 3$, while for frequencies below the peak there are optical depths at which $f<1 / 3$.

Figure $4-14$ is the same as Figure $4-13$, but for a $T_{\text {eff }}=10^{5.6} \mathrm{~K}$ atmosphere computed using $M=40$ angle points. The behavior of $f$ with optical depth is similar to that seen in the $T_{\text {eff }}=10^{6.5} \mathrm{~K}$ atmosphere for the frequencies at or below the peak of the spectrum. For the frequencies above the spectrum peak, the Eddington factor shows that the specific intensity is significantly more anisotropic 
for the lower effective temperature atmosphere. This may be because the frequency independent Thomson scattering is more important in the hotter atmosphere.

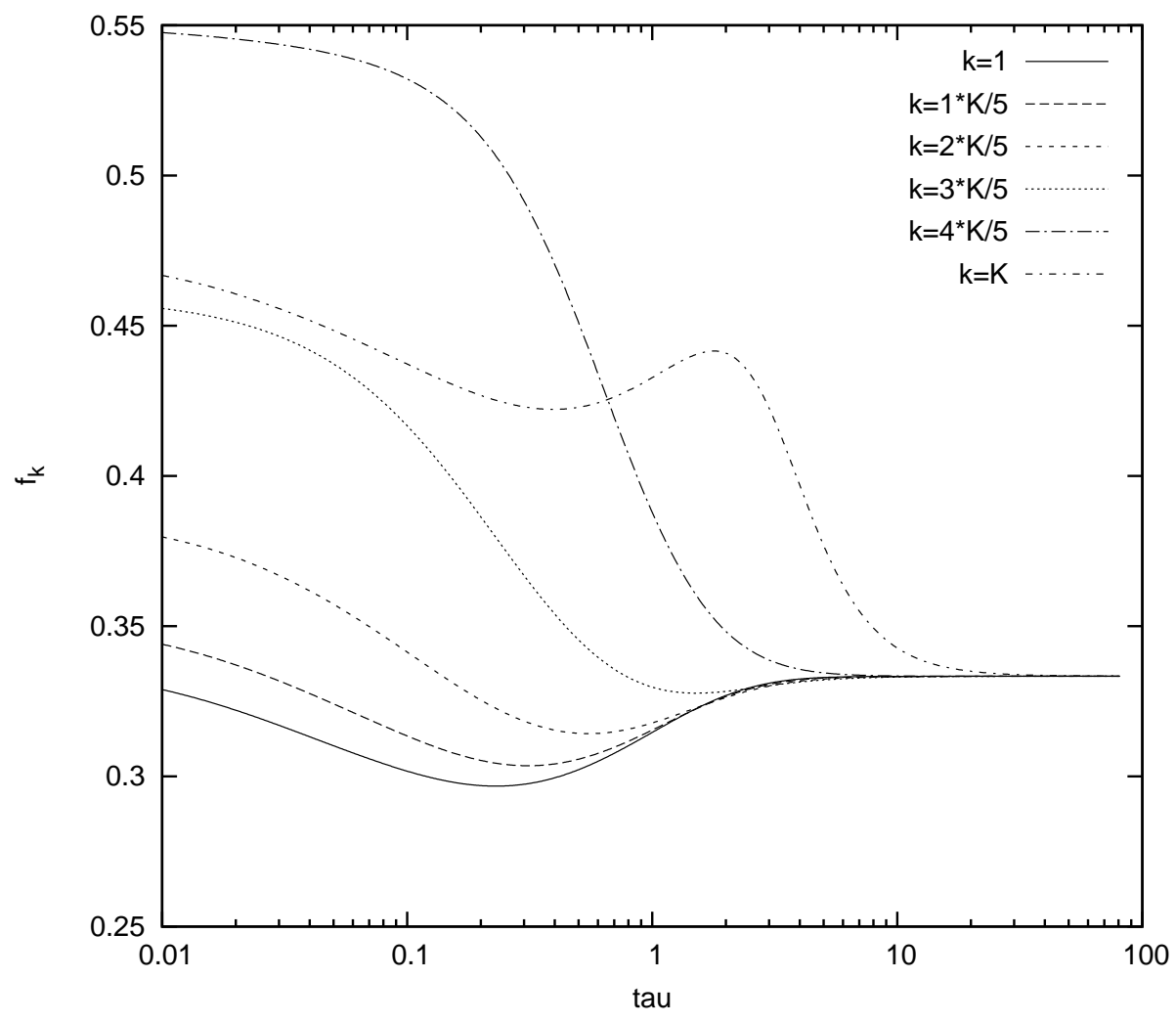

Figure 4-14: Same as Fig. 4-13, but for $T_{\text {eff }}=10^{5.6} \mathrm{~K}$. The behavior of $f$ with optical depth is similar to that seen in the $T_{\text {eff }}=10^{6.5} \mathrm{~K}$ atmosphere for the frequencies at or below the peak of the spectrum. For the frequencies above the spectrum peak, the Eddington factor shows that the specific intensity is significantly more anisotropic for the lower effective temperature atmosphere. 


\section{CHAPTER 5 FUTURE IMPROVEMENTS}

In this section, brief discussion of prospective modifications to the code is given. These modifications could be made in the future to improve McPHAC functionality, its applicability to a particular analysis, or its efficiency.

Collective effects on the opacities. The free-free and Thomson opacities have corrections, due to collective effects, that are of order tens of percent (Boercker 1987; Iglesias \& Rose 1996) which are not included in McPHAC. The magnitude of the effect of these corrections on the output energy spectra has not been estimated, although the percent level differences with Z96 spectra, where some of these effects were considered, indicate that the effect may not be much larger than a few percent. Accurate free-free and Thomson opacities should be included in a future version of the code, preferably through interpolated lookup of detailed monochromatic opacity calculations.

Ionization fraction. The assumption of complete ionization presently limits the application of the McPHAC code to the relatively hot neutron star atmospheres common for qLMXBs. To be able to apply the computed spectra to cooler atmospheres, like those of isolated neutron stars, the code should consider ionization fractions different from unity. The opacity calculation would also have to be updated to account for this. 
Use spectrum computed from temperature correction procedure. In the radiative transfer calculation, full $M \times M$ matrices are inverted $N K$ times, so increasing $M$ comes at a significant computational cost. However, it has been shown that the emergent spectrum can be calculated from the temperature correction, so a computationally advantageous approach may be to optimize the radiative transfer calculation for producing accurate Eddington factors, and then use a high-resolution temperature correction procedure to produce accurate spectra. Optimized depth spacing. McPHAC uses logarithmic spacing of grid points in $y$, both in the radiative transfer calculation and the temperature correction procedure. However, a more optimal spacing of these grid points may exist, so this should be investigated. In any case, the spacing in the radiative transfer calculation should be made in $\tau_{\nu}$, not in $y$, since the discretization of the radiative transfer equation is based on differentials $d \tau_{\nu}$. Furthermore, if the spectrum from the temperature correction procedure is used, and only accurate Eddington factors are required from the radiative transfer calculation, the optimal spacing for accurate Eddington factors may be significantly different from the optimal spacing for an accurate emergent spectrum; optimizing these spacings separately will maximize execution efficiency.

Study of the convergence speed with the number of depth points. The apparent single limiting term in the convergence of the emergent specific intensity with number of depth points in the radiative transfer calculation (cf. Section 4.3) should be investigated. It is possible that convergence can be improved by attempting to minimize this term, and/or by improving the boundary conditions (the bottom 
boundary condition in the radiative transfer calculation is only second order accurate).

Optimized maximum optical depth as a function of photon energy. The radiative transfer calculation at present considers column depth down to a fixed number of optical depths at a given frequency. However, the number of optical depths that need to be considered for a desired accuracy is likely to be strongly frequency dependent, since significant flux at high frequencies for cool atmospheres originates at large optical depth (cf. Section 4.4), while this is not necessarily the case for lower frequencies. Thus, the code could be improved to set the largest optical depth considered at a specific frequency based on the fractional contribution to the flux at the surface from below that depth. Alternately, if the radiative transfer calculation is only used to calculate accurate Eddington factors because the spectrum from the temperature correction procedure is used, the number of optical depths considered could be set based on a target maximum difference between the Eddington factor $f$ and its diffusion limit of $1 / 3$.

Optimized minimum column depth. McPHAC uses a specified column depth range, and then increases the maximum column depth until at least a specified minimum number of optical depths is considered at the largest frequency. A method should be implemented that adjusts the minimum column depth considered, possibly requiring that the column minimum column depth correspond to less than some small threshold optical depth at the lowest frequency.

Optimized number of optical depth points. The present mechanisms in the McPHAC code for adjusting the number of depth points was implemented to 
investigate the convergence of the various parts of the calculation, but this mechanism could be altered to ensure that a sufficient number of depth points is used to achieve a specified accuracy. This could also be extended to the frequency and angle discretizations, so that an optimal number of points is used in each case based on the specified accuracy. Further, the early iterations could be run for a coarser discretization, increasing the number of points in the various variables based on the limiting accuracy due to the size of the temperature correction at that iteration.

Electron heat transport. Zero electron heat transport is an assumption in the present version of the code. The code could be generalized to allow for electron heat transport, which may affect the atmospheric structure enough to significantly affect the emergent spectra. This may also produce a surface gravity dependent effect.

Analytic expressions for out of bounds Gaunt factors. At present, the code uses interpolated gaunt factors from a table. It is possible that gaunt factors outside the range of the table are requested, for which the nearest value on the boundary is used. However, analytic forms could be implemented that give more accurate results outside the range of the table. If the results of detailed monochromatic opacity calculations are implemented (as suggested previously), this would not be necessary, as the Gaunt factor would not itself be needed.

Updating the EOS, and examining the effect of uncertainties in the EOS. The OPAL EOS functions are used to get the equation of state of the atmosphere. The code should be updated to use the most recent version of these functions, and the 
effect of any uncertainties in the equation of state should be investigated, as should the range of validity in temperature and pressure of the functions.

Set initial atmosphere structure based on previously computed atmosphere for similar parameters. For computing emergent spectra on a grid of effective temperatures and surface gravities, it is likely that great computational gains could be made by setting the initial atmospheric structure based on an atmosphere with similar parameters. Doing so could reduce the number of iterations needed for satisfactory convergence.

Computational platform independence. The goal is for the McPHAC code to be platform independent, and to only require a Fortran and $\mathrm{C}$ compiler. However, as of yet, the code has only been tested on the Linux operating system, using the gfortran compiler for both $\mathrm{C}$ and Fortran code. The platform independence should thus be tested and improved.

Implement alternate convergence criteria. The convergence uncertainty is presently controlled by requiring iterations to continue until the maximum fractional temperature change is less than some threshold. An alternative scheme would be to iterate until the effective temperature throughout the atmosphere was uniform to some threshold, and/or until the spectrum changes between iterations by less than some threshold.

Optionally permit isotropic Thomson scattering. A Rybicki type modification (Rybicki 1971) to the Feautrier solution is not possible for anisotropic Thomson scattering, as for the specified $R_{j, j^{\prime}}$ in the present version of McPHAC. However, if the isotropic scattering approximation were to be added, such a modification 
would be possible, and would likely be computationally more efficient and usable in some applications, although sacrificing physical accuracy for other applications. The code to use a Rybicki type solution for isotropic scattering is largely implemented, but remains untested. 


\section{CHAPTER 6 DISCUSSION AND CONCLUSIONS}

The McPHAC v1 code is presented, marking the beginning of an effort to produce model hydrogen atmosphere spectra with uncertainties less than $0.01 \%$ in each spectral bin. The source-code is made available ${ }^{1}$ for public scrutiny, and for further development by members of the scientific community.

An improvement of the present work over previous codes is that in McPHAC, the discretization of the column depth variable in the radiative transfer problem is set independently for each discrete frequency, rather than using the same discretization as in the temperature correction procedure. Using separate discretizations for the two main components of the calculation allows the numerical uncertainty introduced in each component to be studied separately, and allows independent optimization of the two components of the calculation.

A discussion of the numerical convergence of the McPHAC code is given in Section 4.3, and the results indicate that the code is indeed capable of producing spectra with numerical uncertainties less than $0.01 \%$. To constrain other uncertainties in the spectra, however, future work is needed. To this end, a number of possible improvements to the physical assumptions made by the code are suggested

\footnotetext{
${ }^{1}$ http://dualcore.physics.mcgill.ca/McPHAC/
} 
in Chapter 5. Several computational improvements are also suggested there, intended to reduce the computational cost of producing high-accuracy spectra on large grids of parameters.

Of the future developments suggested in Chapter 5 that involve relaxing model assumptions discussed in Chapter 2, the one that should receive the most immediate attention is considering ionization fractions different from unity. The opacity due to neutral hydrogen noticeably affects the spectra of atmospheres with $T_{\text {eff }} \lesssim 10^{5.3} K$ (cf. Z96), but may affect spectra at the $0.01 \%$ level for effective temperatures significantly higher than this, and should thus be included in the calculation. Considering ionization fractions different from unity will require the use of different opacities than those presently in use, so another suggested change that should be implemented concurrently with allowing for other ionization fractions is using monochromatic opacities from detailed opacity calculations. These opacity calculations should consider collective effects (cf. Boercker 1987; Iglesias \& Rose 1996) and ionization fractions different from unity.

Unlike previous non-magnetic hydrogen atmosphere models (Z96; G02; H06), the McPHAC code treats Thomson scattering anisotropically. Spectra computed assuming dipolar Thomson scattering are compared to those computed assuming isotropic scattering, and it is found that there are differences at the level of $10^{-3}$ below $1 \mathrm{keV}$, while the spectra vary by as much as $3 \%$ above $1 \mathrm{keV}$. For the effective temperatures for which a large fraction of the flux is expected above 1 $\mathrm{keV}\left(T_{\text {eff }}=10^{6.2} \mathrm{~K}\right.$ and $\left.T_{\text {eff }}=10^{6.5} \mathrm{~K}\right)$, the differences are at the level of half 
a percent. It is concluded that treating Thomson scattering anisotropically is required if spectra with uncertainties less than $0.01 \%$ are to be produced.

The McPHAC computed spectra are compared in detail to those produced in one of the previous non-magnetic hydrogen atmosphere models (Z96) in Section 4.4. It is found that the $T_{\text {eff }}=10^{5.3} \mathrm{~K}$ and $T_{\text {eff }}=10^{5.6} \mathrm{~K}$ spectra of the previous work predict significantly less flux above $1.5 \mathrm{keV}$ and $3 \mathrm{keV}$, respectively, than do the spectra of the present work. At those frequencies, the spectral flux is down by a factor of $\sim 1 / 30$ from the peak of the spectrum. The cause of the discrepancy is determined to be that the previous work did not consider large enough column depths at those effective temperatures.

The missing flux at high energies in previous work (Z96) is unlikely to significantly affect X-ray spectral fitting for most sources, since the deficit is in a part of the spectrum where the spectral flux is down by a factor of $\sim 1 / 30$ from the peak of the spectrum. However, for sources with $T_{\text {eff }} \lesssim 10^{5.3} \mathrm{~K}$ X-ray absorption may remove much of the observed peak flux, thus making the fitting sensitive to the shape of the spectrum in the range where the flux deficit is seen.

One application for which the missing flux at high energies in previous work (Z96) should be considered, is in X-ray spectral fitting for the isolated neutron star 1RXS J185635.1-375433, which has a best-fit hydrogen atmosphere spectrum with $T_{\text {eff }}=10^{5.2} \mathrm{~K}$ (Pons et al. 2002). This best-fit spectrum is based on an atmosphere model (Pons et al. 2002) for which the largest column depth considered corresponds to 100 optical depths at the Rosseland mean opacity, so the resulting spectra may exhibit the same lack of flux at high energies as discussed 
above. The reason for this is that the opacity drops off quickly with frequency, so the maximum optical depth at the relevant frequencies could be much less than 100. A non-magnetic hydrogen atmosphere has been ruled out as an acceptable model for 1RXS J185635.1-375433 because the best-fit X-ray spectrum overpredicts the optical flux by a factor of 30 (Pons et al. 2002). However, the present work suggests that the X-ray fit may be significantly affected by the failure of the atmosphere models to consider large enough maximum column depths. Thus, the extrapolation to optical flux could be highly uncertain, and the conclusion that a hydrogen atmosphere model is not acceptable is not necessarily sound. The X-ray fitting to 1RXS J185635.1-375433 should therefore be re-visited with model hydrogen atmospheres that are verified to consider a sufficient number of optical depths at large frequencies. The extrapolation to optical flux based on this new fitting can then be used to determine whether the non-magnetic hydrogen atmosphere is an acceptable model for this source. Note that the assumption of complete ionization in the present work limits the McPHAC code accuracy at these low effective temperatures, so if possible another code that considers partial ionization should be used for this detailed investigation of 1RXS J185635.1-375433. 


\section{APPENDIX A RADIATIVE TRANSFER}

The radiative transfer through a plane-parallel atmosphere is described here, based largely on the treatment in previous works (Mihalas 1970, 1978; Rybicki \& Lightman 1979).

The spatial coordinates adopted for the plane-parallel atmosphere are vertical position $z$, polar angle to the outward normal $\theta$, and azimuthal angle $\phi$. The coordinate $z$ is taken to be zero at the surface, and increasingly negative at deeper layers in the atmosphere. In the present application there is azimuthal symmetry, so no dependence on $\phi$ will appear in the radiative transfer. A differential path length $d s$ is taken to be at constant $\phi$, and is thus $d s=d z / \mu$, where $\mu=\cos (\theta)$. Note that the path length $d s$ is always positive.

\section{A.1 Basic Definitions}

\section{A.1.1 Specific Intensity}

The specific intensity $I_{\nu}$ specifies the amount of energy $d E$ carried by rays of radiation that differ infinitesimally from a ray specified by a frequency, position, and direction (no time dependence is considered in the present work):

$$
d E=I_{\nu} d A d \nu d t d \Omega
$$

Here, $I_{\nu}$ is the amount of radiation energy (per unit frequency) between frequen-

cies $[\nu, \nu+d \nu)$ which passes in time $d t$ through an area $d \vec{A}$ (normal to the direction 
of the ray) into a solid angle $d \Omega$. The units of $I_{\nu}$ are thus $\operatorname{ergs~s}^{-1} \mathrm{~cm}^{-2} \mathrm{sr}^{-1} \mathrm{~Hz}^{-1}$. Note that $I_{\nu}$ depends on position, direction, and frequency.

\section{A.1.2 Flux}

The flux is the net rate of energy flow in a direction $\vec{n}$, and is related to the specific intensity through

$$
F_{\nu}=\int I_{\nu} \cos \xi d \Omega
$$

where $\xi$ is the angle between the direction of $I_{\nu}$ and the direction $\vec{n}$.

\section{A.1.3 Mean Specific Intensity}

The mean specific intensity $J_{\nu}$ is the angle-average specific intensity:

$$
J_{\nu}=\frac{\int I_{\nu} d \Omega}{4 \pi}
$$

For isotropic radiation, $J_{\nu}=I_{\nu}$.

\section{A.1.4 True Absorption}

One way radiation can interact with matter is through photons being removed from the radiation field through true absorption, where the photon energy is deposited as thermal energy in the matter rather than being immediately reemitted. The change in the specific intensity due to absorption by matter of density $\rho\left(\mathrm{g} \mathrm{cm}^{-3}\right)$ and opacity $\kappa_{\nu}\left(\mathrm{cm}^{2} \mathrm{~g}^{-1}\right)$, in a path length $d s(\mathrm{~cm})$, is given by

$$
d I_{\nu, \mathrm{abs}}=-\kappa_{\nu} \rho I_{\nu} d s
$$




\section{A.1.5 Emission}

Material may also emit radiation. Using Kirchoff's law for thermal emission, the change in specific intensity due to emission can be written as

$$
d I_{\nu, \mathrm{em}}=\kappa_{\nu} \rho B_{\nu} d s
$$

where $B_{\nu}$ is blackbody intensity.

\section{A.1.6 Thomson Scattering}

Scattering is treated as absorption with immediate re-emission of radiation, and is assumed to be coherent in the present work. It is assumed here that the Thomson scattering is anisotropic, as is the usual description for Thomson scattering, but which has not been employed in previous $\mathrm{H}$ atmosphere calculations (Z96, G02, H06). The absorption opacity is taken to be the reduced Thomson opacity $\sigma_{\mathrm{T}, \mathrm{r}}$, which for pure fully ionized hydrogen is the standard Thomson cross section $\sigma_{T}$ (in $\mathrm{cm}^{-2}$ ) divided by $m_{p}+m_{e}$ (the proton mass plus the electron mass). The absorbed photons are taken to be re-emitted according to the differential opacity $d \sigma_{\mathrm{T}, \mathrm{r}} / d \Omega$, so that the total change in the specific intensity due to scattering is

$$
d I_{\nu, \text { scatt }}=\left(-\sigma_{\mathrm{T}, \mathrm{r}} I_{\nu}+\int I_{\nu} \frac{d \sigma_{\mathrm{T}, \mathrm{r}}}{d \Omega} d \Omega\right) \rho d s .
$$

\section{A.1.7 Total Absorption Opacity}

Given both true absorption and scattering, Equations A.4 and A.6 motivate the definition of the total absorption opacity:

$$
k_{\nu}=\kappa_{\nu}+\sigma_{\mathrm{T}, \mathrm{r}} .
$$




\section{A.1.8 Effective Optical Depth}

The total absorption opacity can be used to define the effective optical depth $\tau_{\nu, \text { eff }}$ of a path length $s^{\prime}$ between two points:

$$
\tau_{\nu, \text { eff }}=\int_{0}^{s^{\prime}} k_{\nu} \rho d s
$$

Further, the effective normal optical depth $\tau_{\nu}$ is taken to be the effective optical depth from the surface in the direction $\mu=-1$, so that

$$
d \tau_{\nu}=-k_{\nu} \rho d z
$$

\section{A.1.9 Radiative Transfer Equation}

Combining Equations A.4, A.5, and A.6 gives the total change in the specific intensity due to absorption, emission, and scattering:

$$
d I_{\nu}=\left(-\kappa_{\nu} I_{\nu}+\kappa_{\nu} B_{\nu}-\sigma_{\mathrm{T}, \mathrm{r}} I_{\nu}+\int I_{\nu} \frac{d \sigma_{\mathrm{T}, \mathrm{r}}}{d \Omega} d \Omega\right) \rho d s
$$

This equation can be re-written to the more standard form of the transfer equation,

$$
\frac{\mu}{k_{\nu} \rho} \frac{d I_{\nu}}{d z}=-\frac{\kappa_{\nu}}{k_{\nu}}\left(I_{\nu}-B_{\nu}\right)-\frac{\sigma_{\mathrm{T}, \mathrm{r}}}{k_{\nu}}\left(I_{\nu}-\frac{1}{\sigma_{\mathrm{T}, \mathrm{r}}} \int I_{\nu} \frac{d \sigma_{\mathrm{T}, \mathrm{r}}}{d \Omega} d \Omega\right)
$$

where the previously presented relation $d s=d z / \mu$ has been used, and the equation divided by the total absorption opacity. 


\section{A.1.10 Column Depth}

The column depth $y\left(\mathrm{~g} \mathrm{~cm}^{-2}\right)$ is defined as the amount of material vertically above a depth $z$ in the atmosphere, so that

$$
d y=-\rho d z
$$

\section{A.1.11 Dimensionless Scattering Albedo}

The fraction of the total absorption opacity due to scattering is the dimensionless scattering albedo:

$$
\rho_{\nu}=\frac{\sigma_{\mathrm{T}, \mathrm{r}}}{\kappa_{\nu}+\sigma_{\mathrm{T}, \mathrm{r}}}=\frac{\sigma_{\mathrm{T}, \mathrm{r}}}{k_{\nu}}
$$

\section{A.1.12 Source Function}

The source function $S_{\nu}(y, \mu)$ is a collection of the source terms from the RHS of Equation A.11:

$$
S_{\nu}(y, \mu)=\left(1-\rho_{\nu}\right) B_{\nu}(T(y))+\rho_{\nu} \frac{1}{\sigma_{\mathrm{T}, \mathrm{r}}} \int_{-1}^{1} I_{\nu} \frac{d \sigma_{\mathrm{T}, \mathrm{r}}}{d \mu^{\prime}}\left(\mu, \mu^{\prime}\right) d \mu^{\prime}
$$

Here, the differential scattering opacity has been re-written in terms of the ingoing photon direction $\mu^{\prime}$ and outgoing direction $\mu$.

Using the definition of the source function, dimensionless scattering albedo, and column depth, Equation A.11 can be re-written as

$$
\frac{\mu}{k_{\nu}} \frac{d I_{\nu}(y, \mu)}{d y}=I_{\nu}(y, \mu)-S_{\nu}(y, \mu)
$$




\section{A.2 Second Order Form of the Transfer Equation}

This section shows how to re-write the transfer equation as a second order differential equation, which is the form in which it is most commonly solved. The following derivation differs from the standard approach (e.g. Mihalas 1978) in that it has been modified to allow for anisotropic scattering.

For $\mu>0$, one makes the definitions

$$
P_{\nu}(y, \mu) \equiv \frac{1}{2}\left[I_{\nu}(y, \mu)+I_{\nu}(y,-\mu)\right]
$$

and

$$
R_{\nu}(y, \mu) \equiv \frac{1}{2}\left[I_{\nu}(y, \mu)-I_{\nu}(y,-\mu)\right]
$$

It is then noted that Equation A.15 for negative values of $\mu$ can be re-written as

$$
-\frac{\mu}{k_{\nu}} \frac{d I_{\nu}(y,-\mu)}{d y}=I_{\nu}(y,-\mu)-S_{\nu}(y,-\mu)
$$

where in this case $\mu$ is positive.

Equations A.15 and A.18 are added to give

$$
\frac{\mu}{k_{\nu}} \frac{d R_{\nu}(y, \mu)}{d y}=P_{\nu}(y, \mu)-S_{\nu}(y, \mu)
$$

and subtracted to give

$$
\frac{\mu}{k_{\nu}} \frac{d P_{\nu}(y, \mu)}{d y}=R_{\nu}(y, \mu) .
$$

Substituting Equation A.20 into Equation A.19 gives the second order form of the transfer equation:

$$
\frac{\mu^{2}}{k_{\nu}} \frac{d}{d y}\left(\frac{1}{k_{\nu}} \frac{d P_{\nu}(y, \mu)}{d y}\right)=P_{\nu}(y, \mu)-S_{\nu}(y, \mu) .
$$


To solve Equation A.21, the source function must first be written in terms of $P_{\nu}$, and the angle integral discretized to create a system of equations to be solved. To do this, note that

$$
\int_{-1}^{1} I_{\nu} \frac{d \sigma_{\mathrm{T}, \mathrm{r}}}{d \mu^{\prime}}\left(\mu, \mu^{\prime}\right) d \mu^{\prime}=2 \int_{0}^{1} P_{\nu}\left(y, \mu^{\prime}\right) \frac{d \sigma_{\mathrm{T}, \mathrm{r}}}{d \mu^{\prime}}\left(\mu, \mu^{\prime}\right) d \mu^{\prime},
$$

and approximate the integral using a Gauss-Legendre quadrature sum with weights $a_{j^{\prime}}$ :

$$
\int_{0}^{1} P_{\nu}\left(y, \mu^{\prime}\right) \frac{d \sigma_{\mathrm{T}, \mathrm{r}}}{d \mu^{\prime}}\left(\mu, \mu^{\prime}\right) d \mu^{\prime} \approx \sum_{j^{\prime}=1}^{M} P_{\nu}\left(y, \mu_{j^{\prime}}\right) \frac{d \sigma_{\mathrm{T}, \mathrm{r}}}{d \mu^{\prime}}\left(\mu, \mu_{j^{\prime}}\right) a_{j^{\prime}} .
$$

The source function can thus be re-written as

$$
S_{\nu}(y, \mu)=\left(1-\rho_{\nu}\right) B_{\nu}(T(y))+2 \rho_{\nu} \sum_{j^{\prime}=1}^{M} P_{\nu}\left(y, \mu_{j^{\prime}}\right) \frac{d \sigma_{\mathrm{T}, \mathrm{r}}}{d \mu^{\prime}}\left(\mu, \mu_{j^{\prime}}\right) a_{j^{\prime}},
$$

which allows the second order transfer equation (Equation A.21) to be written as $\frac{\mu^{2}}{k_{\nu}} \frac{d}{d y}\left(\frac{1}{k_{\nu}} \frac{d P_{\nu}(y, \mu)}{d y}\right)=P_{\nu}(y, \mu)-\left(1-\rho_{\nu}\right) B_{\nu}(T(y))-2 \rho_{\nu} \sum_{j^{\prime}=1}^{M} P_{\nu}\left(y, \mu_{j^{\prime}}\right) \frac{d \sigma_{\mathrm{T}, \mathrm{r}}}{d \mu^{\prime}}\left(\mu, \mu_{j^{\prime}}\right) a_{j^{\prime}}$.

\section{A.3 Computing the Specific Intensities}

Once $P_{\nu}(y, \mu)$ has been found by solution of Equation A.25, $R_{\nu}(y, \mu)$ can be calculated using Equation A.20. The specific intensity can then recovered using

$$
I_{\nu}(y, \mu)=P_{\nu}(y, \mu)+R_{\nu}(y, \mu) .
$$


However, at the surface the specific intensity is given by

$$
\begin{array}{lcl}
I_{\nu}(0, \mu)= & 2 P_{\nu}(0, \mu) & , \mu \geq 0 \\
I_{\nu}(0, \mu)= & 0 & , \mu<0,
\end{array}
$$

so the emergent specific intensity at the surface can be computed directly from $P_{\nu}(0, \mu)$ 


\section{APPENDIX B Observed Spectrum}

Once the specific intensity at the surface at emission angle $\alpha$ and frequency $\nu_{0}, I_{0}\left(\nu_{0}, \mu\right)$, is known $(\mu=\cos \alpha)$, the spectral flux $F(\nu)$ observed at a frequency $\nu$ by an observer far from the neutron star should be computed. This section derives an expression for $F(\nu)$ in terms of $I_{0}\left(\nu_{0}, \mu\right)$, largely inspired by a discussion in the appendix of a previous work (Beloborodov 2002). The main difference between the present discussion and that in the previous work is that the present work considers the specific intensity as a function of frequency (rather than the frequency-integrated specific intensity), and the present work uses the added assumption that $I_{0}\left(\nu_{0}, \mu\right)$ does not vary across the neutron star surface.

Consider Schwartzschild coordinates $(t, r, \theta, \phi)$ centered on a non-rotating neutron star of mass $M$ and radius $R$. The shape of a photon orbit in the plane $\theta=\pi / 2$ is determined by the impact parameter $b$ (Misner et al. 1973). To an

observer at $r \geq R$, the angle $\xi$ between the radial direction and the photon path is given by (Pechenick et al. 1983)

$$
\sin \xi=\frac{b}{r} \sqrt{1-\frac{r_{g}}{r}},
$$

where $r_{g}=2 G M / c^{2}$ is the Schwartzschild radius. This expression can be used to relate the emission angle $\alpha$ at the surface to the impact parameter $b$, by replacing 
$\xi$ with $\alpha$ and $r$ with $R$. The maximum impact parameter is (Pechenick et al. 1983)

$$
b_{\max }=\frac{R}{\sqrt{1-\frac{r_{g}}{r}}} .
$$

Thus, to an observer at distance $D \gg R$, the small angle approximation can be used to give $\xi=b / D$ (also neglecting the $r_{g} / D<R / D \ll 1$ term).

To the observer at $D$, the radiation from a (visible) surface area element $d S=R^{2} \sin \phi d \phi d \theta$ is observed for impact parameters in the range $[b, b+d b)$ (cf. Beloborodov 2002), subtending solid angle $d \Omega=\sin \xi d \xi d \phi=b d b d \phi / D^{2}$. The amount of flux observed at frequency $\nu$ from that surface area element is thus $d F(\nu)=I(\nu, \xi) \sin \xi d \xi d \phi=I(\nu, b) b d b d \phi / D^{2}$. The specific intensity at the observer, $I(\nu, b)$, is related to the specific intensity at the surface, $I_{0}\left(\nu_{0}, b\right)$, through the relations $I(\nu, b) / \nu^{3}=I_{0}\left(\nu_{0}, b\right) / \nu_{0}^{3}$ and $\nu / \nu_{0}=\sqrt{1+r_{g} / R}$ (Misner et al. 1973) $)^{1}$. Thus, the flux observed at frequency $\nu$ is

$$
d F(\nu, b)=I_{0}\left(\nu_{0}, b\right)\left(1-\frac{r_{g}}{R}\right)^{3 / 2} \frac{b d b d \phi}{D^{2}} .
$$

Noting that equation (B.1) can be used to write $b d b=R^{2} /\left(1-r_{g} / R\right) \mu d \mu$, the expression becomes

$$
d F(\nu, b)=I_{0}\left(\nu_{0}, \alpha\right) \sqrt{1-\frac{r_{g}}{R}} \frac{R^{2}}{D^{2}} \mu d \mu d \phi,
$$

\footnotetext{
${ }^{1}$ For the frequency integrated specific intensity, the corresponding relation is $I / \nu^{4}=I_{0} / \nu_{0}^{4}$ (Beloborodov 2002).
} 
which can be integrated to give

$$
F(\nu)=2 \pi \int_{0}^{1} I_{0}\left(\nu_{0}, \alpha\right) \mu d \mu \sqrt{1-\frac{r_{g}}{R}} \frac{R^{2}}{D^{2}}=F_{0}\left(\nu_{0}\right) \sqrt{1-\frac{r_{g}}{R}} \frac{R^{2}}{D^{2}} .
$$

Note that this result is only valid when $I_{0}\left(\nu_{0}, \alpha\right)$ is the same for every point on the surface.

The expression in equation (B.5) allows the spectrum seen by an observer far from the neutron star to be calculated from the emergent spectrum. 


\section{APPENDIX C TEMPERATURE CORRECTION SOLUTION}

\section{C.1 Temperature correction}

The temperature correction scheme used in the present work is based on that used in previous work (Z96), and is described in detail in this section.

First, the second order transfer equation is integrated over $\mu$ to give

$\frac{1}{k_{\nu}} \frac{d}{d y}\left(\frac{1}{k_{\nu}} \frac{d}{d y} \int_{0}^{1} \mu^{2} P_{\nu}(y, \mu) d \mu\right)=J_{\nu}-\left(1-\rho_{\nu}\right) B_{\nu}-\frac{2 \rho_{\nu}}{\sigma_{\mathrm{T}, \mathrm{r}}} \int_{0}^{1} d \mu \int_{0}^{1} d \mu^{\prime} P_{\nu}\left(y, \mu^{\prime}\right) \frac{d \sigma_{\mathrm{T}, \mathrm{r}}}{d \mu^{\prime}}\left(\mu, \mu^{\prime}\right)$.

The last term in the above equation must reduce to $\rho_{\nu} J_{\nu}$ since the total angle integrated specific intensity emitted through scattering must equal that absorbed. Using this fact, and the definition of the Eddington factor $f_{\nu}=J_{\nu}^{-1} \int_{0}^{1} \mu^{2} P_{\nu}(y, \mu) d \mu$, Equation C.1 reduces to

$$
\frac{1}{k_{\nu}} \frac{d}{d y}\left(\frac{1}{k_{\nu}} \frac{d}{d y}\left(f_{\nu} J_{\nu}\right)\right)=\left(J_{\nu}-B_{\nu}(T(y))\right)\left(1-\rho_{\nu}\right)
$$


To proceed, it is noted that the energy flux at a given frequency can be written as $F_{\nu}=\frac{4 \pi}{k_{\nu}} \frac{d}{d y}\left(f_{\nu} J_{\nu}\right)$. To show this, simply write

$$
\begin{aligned}
\frac{4 \pi}{k_{\nu}} \frac{d}{d y}\left(f_{\nu} J_{\nu}\right) & =\frac{4 \pi}{k_{\nu}} \frac{d}{d y} \int_{0}^{1} \mu^{2} P_{\nu}(y, \mu) d \mu \\
& =\frac{4 \pi}{k_{\nu}} \int_{0}^{1} \mu^{2} \frac{d P_{\nu}(y, \mu)}{d y} d \mu \\
& =4 \pi \int_{0}^{1} \mu R_{\nu}(y, \mu) d \mu \\
& =2 \pi \int_{0}^{1} \mu I_{\nu}(y, \mu) d \mu-2 \pi \int_{0}^{1} \mu I_{\nu}(y,-\mu) d \mu \\
& =2 \pi \int_{-1}^{1} \mu I_{\nu}(y, \mu) d \mu,
\end{aligned}
$$

which is the flux as defined previously.

Inserting the result $F_{\nu}=\frac{4 \pi}{k_{\nu}} \frac{d}{d y}\left(f_{\nu} J_{\nu}\right)$ into Equation C.2, multiplying by $k_{\nu}$, and integrating over all frequencies gives

$$
\frac{1}{4 \pi} \frac{d}{d y} \int_{0}^{\infty} F_{\nu} d \nu=\int_{0}^{\infty}\left(J_{\nu}-B_{\nu}(T(y))\right) \kappa_{\nu} d \nu
$$

since $\left(1-\rho_{\nu}\right) k_{\nu}=\kappa_{\nu}$. In radiative equilibrium, the energy flux through every depth of the atmosphere will be the same. Thus, the LHS of the above equation vanishes, so the RHS must also equal zero. Taking the radiative equilibrium temperature profile to be $T(y)=T_{0}(y)+\Delta T(y)$, where $T_{0}(y)$ is the present best guess temperature profile and $\Delta T(y)$ is a correction to this profile, the blackbody function can be expanded to first order in a Taylor Series: $B(T)=$ $B\left(T_{0}\right)+d B / d T \Delta T$. Together with Equation C.8, this gives the expression

$$
0=\int_{0}^{\infty}\left(J_{\nu}-B_{\nu}\left(T_{0}\right)-\frac{d B_{\nu}}{d T} \Delta T\right) \kappa_{\nu} d \nu
$$


which can be solved for the temperature correction:

$$
\Delta T=\frac{\int_{0}^{\infty}\left(J_{\nu}-B_{\nu}\left(T_{0}\right)\right) \kappa_{\nu} d \nu}{\int_{0}^{\infty} \frac{d B_{\nu}}{d T} \kappa_{\nu} d \nu}
$$

It is important to stress that the temperature correction computed in this way will satisfy constant energy flux through the atmosphere, since this was assumed in equating the RHS of Equation C.8 to zero.

To find $J_{\nu}$, and thus $\Delta T$, the equation to solve comes from combining Equation C.10 with Equation C.2 with the Taylor expansion for $B(T)$ above, to give:

$$
\frac{1}{k_{\nu}} \frac{d}{d y}\left(\frac{1}{k_{\nu}} \frac{d}{d y}\left(f_{\nu} J_{\nu}\right)\right)=\left(J_{\nu}-B_{\nu}\left(T_{0}\right)-\frac{d B_{\nu}}{d T} \frac{\int_{0}^{\infty}\left(J_{\nu}-B_{\nu}\left(T_{0}\right)\right) \kappa_{\nu} d \nu}{\int_{0}^{\infty} \frac{d B_{\nu}}{d T} \kappa_{\nu} d \nu}\right)\left(1-\rho_{\nu}\right) .
$$

To solve this equation, the $f_{\nu}$ computed by solving the full angular radiative transfer problem is used. By using a fixed $f_{\nu}$ this equation can be solved using a modified (by removing dependence on an angular variable) Rybicki approach (Rybicki 1971), which will give a corrected temperature profile. The full angular radiative transfer problem can then be solved for the corrected temperature profile, which will give a new $f_{\nu}$, which will allow a new temperature correction to be calculated. Iterations can be repeated until satisfactory convergence.

\section{C.2 Discretization}

The discretization of Equation C.11 is described here in full. An alternate approach would be to use a discretization based on the hermitian method discussed in previous work (Auer 1976), which may be more accurate, but this is left for future work. 
The first thing to do is to re-write the LHS of Equation C.11 using the expression $d \tau_{\nu}=k_{\nu} d y$, which gives

$$
\frac{\mu^{2}}{k_{\nu}} \frac{d}{d y}\left(\frac{1}{k_{\nu}} \frac{d}{d y}\left(f_{\nu} J_{\nu}\right)\right)=\mu^{2} \frac{d}{d \tau_{\nu}}\left(\frac{d}{d \tau_{\nu}}\left(f_{\nu} J_{\nu}\right)\right) .
$$

Then, the derivatives at some discrete depth point $y_{i}$ are approximated using

$$
\left(\frac{d\left(f_{\nu} J_{\nu}\right)}{d \tau_{\nu}}\right)_{i \pm 1 / 2} \approx \frac{\left(\Delta\left(f_{\nu} J_{\nu}\right)\right)_{i \pm 1 / 2}}{\left(\Delta \tau_{\nu}\right)_{i \pm 1 / 2}}
$$

and

$$
\mu^{2} \frac{d}{d \tau}\left(\frac{d\left(f_{\nu} J_{\nu}\right)}{d \tau}\right) \approx \mu^{2} \frac{\left(\Delta\left(\frac{\left.d\left(f_{\nu} J_{\nu}\right)\right)}{d \tau_{\nu}}\right)\right)_{i}}{\left(\Delta \tau_{\nu}\right)_{i}} \approx \mu^{2} \frac{\frac{\left(\Delta\left(f_{\nu} J_{\nu}\right)\right)_{i+1 / 2}}{\left(\Delta \tau_{\nu}\right) i_{i+1 / 2}}-\frac{\left(\Delta\left(f_{\nu} J_{\nu}\right)\right)_{i-1 / 2}}{\left(\Delta \tau_{\nu}\right)_{i-1 / 2}}}{\frac{1}{2}\left(\left(\Delta \tau_{\nu}\right)_{i+1 / 2}+\left(\Delta \tau_{\nu}\right)_{i-1 / 2}\right)},
$$

where $i \pm 1 / 2$ is used to mean evaluation at the midpoints between $y_{i}$ and its nearest neighbors. Plugging in

$$
\left(\Delta\left(f_{\nu} J_{\nu}\right)\right)_{i \pm 1 / 2}= \pm\left(\left(f_{\nu} J_{\nu}\right)\left(y_{i \pm 1}\right)-\left(f_{\nu} J_{\nu}\right)\left(y_{i}\right)\right),
$$

and the approximations

$$
\left(\Delta \tau_{\nu}\right)_{i \pm 1 / 2} \approx k_{\nu}\left(y_{i \pm 1 / 2}\right)(\Delta y)_{i \pm 1 / 2}
$$

and

$$
k_{i \pm 1 / 2} \approx \frac{1}{2}\left(k_{i}+k_{i \pm 1}\right),
$$

gives the full discrete expression

$$
\mu^{2} \frac{d^{2}}{d \tau_{\nu}^{2}}\left(f_{\nu} J_{\nu}\right) \approx \frac{\left(\frac{\left(f_{\nu} J_{\nu}\right)\left(y_{i+1}\right)-\left(f_{\nu} J_{\nu}\right)\left(y_{i}\right)}{\frac{1}{2}\left[k_{\nu}\left(y_{i+1}\right)+k_{\nu}\left(y_{i}\right)\right]\left(y_{i+1}-y_{i}\right)}-\frac{\left(f_{\nu} J_{\nu}\right)\left(y_{i}\right)-\left(f_{\nu} J_{\nu}\right)\left(y_{i-1}\right)}{\frac{1}{2}\left[k_{\nu}\left(y_{i}\right)+k_{\nu}\left(y_{i-1}\right)\right]\left(y_{i}-y_{i-1}\right)}\right)}{\frac{1}{2}\left[\frac{1}{2}\left(k_{\nu}\left(y_{i+1}\right)+k_{\nu}\left(y_{i}\right)\right)\left(y_{i+1}-y_{i}\right)+\frac{1}{2}\left(k_{\nu}\left(y_{i}\right)+k_{\nu}\left(y_{i-1}\right)\right)\left(y_{i}-y_{i-1}\right)\right]} .
$$


Also approximating the integrals in Equation C.11 as discrete sums, that equation thus becomes

$$
\begin{gathered}
-\frac{8}{\pi \Delta_{1}}\left(f_{k} J_{k}\right)\left(y_{i-1}\right) \\
+\left[\frac{1-\rho_{k}}{f_{k}\left(y_{i}\right)}+\frac{8}{\pi \Delta_{1}}+\frac{8}{\pi \Delta_{2}}\right]\left(f_{k} J_{k}\right)\left(y_{i}\right) \\
-\frac{d B_{k}}{d T} \frac{\sum_{k^{\prime}=1}^{K} J_{k^{\prime}} \kappa_{k^{\prime}} b_{k^{\prime}}}{\sum_{k^{\prime}=1}^{K} \frac{d B_{k^{\prime}}}{d T} \kappa_{k^{\prime}} b_{k^{\prime}}}\left(1-\rho_{k}\right) \\
-\frac{8}{\pi \Delta_{2}}\left(f_{k} J_{k}\right)\left(y_{i+1}\right)=\left(B_{k}\left(T\left(y_{i}\right)\right)-\frac{d B_{k}}{d T} \frac{\sum_{k^{\prime}=1}^{K} B_{k^{\prime}}\left(T_{0}\right) \kappa_{k^{\prime}} b_{k^{\prime}}}{\sum_{k^{\prime}=1}^{K} \frac{d B_{k^{\prime}}}{d T} \kappa_{k^{\prime}} b_{k^{\prime}}}\right)\left(1-\rho_{k}\right),
\end{gathered}
$$

where the subscript $k$ is used to indicate a discrete frequency point (not to be confused with the total absorption opacity $k_{\nu}$ ), and the following definitions have been made to simplify the notation:

$$
\begin{aligned}
\Delta_{1} & =\left[k_{\nu}\left(y_{i}\right)+k_{\nu}\left(y_{i-1}\right)\right]\left(y_{i}-y_{i-1}\right) \\
\Delta_{2} & =\left[k_{\nu}\left(y_{i+1}\right)+k_{\nu}\left(y_{i}\right)\right]\left(y_{i+1}-y_{i}\right) \\
\pi & =\Delta_{1}+\Delta_{2} .
\end{aligned}
$$

Proceeding as in the Feautrier method (Feautrier 1964), the above equation can be written in the form

$$
\mathbf{A}_{\mathbf{i}} \mathbf{J}_{\mathbf{i}-\mathbf{1}}+\mathbf{B}_{\mathbf{i}} \mathbf{J}_{\mathbf{i}}+\mathbf{C}_{\mathbf{i}} \mathbf{J}_{\mathbf{i}+\mathbf{1}}=\mathbf{Q}_{\mathbf{i}}
$$


which gives rise to the tri-diagonal grand matrix equation

$$
\left(\begin{array}{cccccc}
\mathbf{B}_{1} & \mathbf{C}_{1} & 0 & \ldots & \ldots & 0 \\
\mathbf{A}_{2} & \mathbf{B}_{2} & \mathbf{C}_{2} & 0 & \ldots & \vdots \\
0 & \mathbf{A}_{3} & \mathbf{B}_{3} & \ddots & \ddots & \vdots \\
\vdots & 0 & \ddots & \ddots & \ddots & 0 \\
\vdots & \vdots & \ddots & \ddots & \ddots & \mathbf{C}_{\mathrm{N}-1} \\
0 & \ldots & \ldots & 0 & \mathbf{A}_{\mathrm{N}} & \mathbf{B}_{\mathrm{N}}
\end{array}\right)\left(\begin{array}{c}
\mathbf{J}_{1} \\
\mathbf{J}_{2} \\
\mathbf{J}_{3} \\
\vdots \\
\vdots \\
\mathbf{J}_{\mathrm{N}}
\end{array}\right)=\left(\begin{array}{c}
\mathrm{Q}_{1} \\
\mathbf{Q}_{2} \\
\mathrm{Q}_{3} \\
\vdots \\
\vdots \\
\mathbf{Q}_{\mathrm{N}}
\end{array}\right) .
$$

Here, $\mathbf{A}_{\mathbf{i}}, \mathbf{B}_{\mathbf{i}}$, and $\mathbf{C}_{\mathbf{i}}$ are $K \times K$ matrices, $\mathbf{J}_{\mathbf{i}}$ and $\mathbf{Q}_{\mathbf{i}}$ are vectors of dimension $K$ (the number of frequency points), and $N=N_{\text {temp }}$ (the number of depth points).

The explicit matrix elements are given by

$$
\begin{aligned}
\left(A_{i}\right)_{k k^{\prime}} & =-\frac{8 f_{k}\left(y_{i-1}\right)}{\pi \Delta_{1}} \delta_{k, k^{\prime}} \\
\left(C_{i}\right)_{k k^{\prime}} & =-\frac{8 f_{k}\left(y_{i+1}\right)}{\pi \Delta_{2}} \delta_{k, k^{\prime}} \\
\left(B_{i}\right)_{k k^{\prime}} & =\left[\frac{1-\rho_{k}}{f_{k}\left(y_{i}\right)}+\frac{8}{\pi \Delta_{1}}+\frac{8}{\pi \Delta_{2}}\right] f_{k}\left(y_{i}\right) \delta_{k, k^{\prime}}-\frac{d B_{k}}{d T} \frac{k_{k^{\prime}} b_{k^{\prime}}\left(1-\rho_{k}\right)}{\sum_{k^{\prime \prime}=1}^{K} \frac{d B_{k^{\prime \prime}}}{d T} k_{k^{\prime \prime}} b_{k^{\prime \prime}}} \\
\left(Q_{i}\right)_{k} & =\left(B_{k}\left(T\left(y_{i}\right)\right)-\frac{d B_{k}}{d T} \frac{\sum_{k^{\prime}=1}^{K} B_{k^{\prime}}\left(T_{0}\right) k_{k^{\prime}} b_{k^{\prime}}}{\sum_{k^{\prime}=1}^{K} \frac{d B_{k^{\prime}}}{d T} k_{k^{\prime}} b_{k^{\prime}}}\right)\left(1-\rho_{k}\right)
\end{aligned}
$$

for $1<i<N_{\text {temp }}$, where $\delta_{k, k^{\prime}}$ is the Kronecker delta. The $i=1$ and $i=N_{\text {temp }}$ matrices are specified by the boundary conditions, which are discussed in Section C.4. 
If the boundary conditions are specified, the matrix equation can now be solved using the Feautrier method (Feautrier 1964). However, a less computationally intensive method of solution is possible: the Rybicki method (Rybicki 1971). This method is now described.

\section{C.3 Rybicki Method}

The Rybicki method (Rybicki 1971) involves re-structuring the matrix problem so that most of the matrices that have to be inverted are tri-diagonal matrices, which are less computationally demanding to invert than full matrices. This section describes the specific application of the previously described method (Rybicki 1971) to the temperature correction problem.

Rather than being grouped into $N_{\text {temp }}$ vectors of dimension $K$, the values of the mean specific intensity are now written as the $K$ column vectors

$$
\mathbf{J}_{\mathbf{k}}=\left(J_{k, 1}, J_{k, 2}, J_{k, 3}, \ldots, J_{k, N}\right)^{T} .
$$

In addition, one defined the vector $\overline{\mathbf{J}}$, which has elements

$$
(\bar{J})_{i}=\frac{\sum_{k^{\prime}=1}^{K} J_{k^{\prime}} \kappa_{k^{\prime}} b_{k^{\prime}}}{\sum_{k^{\prime}=1}^{K} \frac{d B_{k^{\prime}}}{d T} \kappa_{k^{\prime}} b_{k^{\prime}}} .
$$

Equation C.23 can then be re-written in the form

$$
\mathbf{T}_{\mathbf{k}} \mathbf{J}_{\mathrm{k}}+\mathbf{U}_{\mathrm{k}} \overline{\mathbf{J}}=\mathbf{K}_{\mathrm{k}}
$$


where the matrix elements are given by

$$
\begin{aligned}
\left(T_{k}\right)_{i, i-1} & =-\frac{\left.8 f_{k}\left(y_{i-1}\right)\right)}{\pi \Delta_{1}} \\
\left(T_{k}\right)_{i, i+1} & =-\frac{8 f_{k}\left(y_{i+1}\right)}{\pi \Delta_{2}} \\
\left(T_{k}\right)_{i, i} & =\left[\frac{1-\rho_{k}}{f_{k}\left(y_{i}\right)}+\frac{8}{\pi \Delta_{1}}+\frac{8}{\pi \Delta_{2}}\right] f_{k}\left(y_{i}\right) \\
\left(U_{k}\right)_{i, i} & =-\frac{d B_{k}}{d T}\left(1-\rho_{k}\right) \\
\left(K_{k}\right)_{i} & =\left(B_{k}\left(T\left(y_{i}\right)\right)-\frac{d B_{k}}{d T} \frac{\sum_{k^{\prime}=1}^{K} B_{k^{\prime}}\left(T_{0}\right) \kappa_{k^{\prime}} b_{k^{\prime}}}{\sum_{k^{\prime}=1}^{K} \frac{d B_{k^{\prime}}}{d T} \kappa_{k^{\prime}} b_{k^{\prime}}}\right)\left(1-\rho_{k}\right),
\end{aligned}
$$

for $1<i<N_{\text {temp. }}$ All other elements of $\mathbf{T}_{\mathbf{k}}$ and $\mathbf{U}_{\mathbf{k}}$ are zero, except those that are specified in the boundary conditions (cf. Section C.4).

The corresponding grand matrix equation is

$$
\left(\begin{array}{ccccc}
\mathbf{T}_{1} & 0 & \cdots & 0 & \mathbf{U}_{1} \\
0 & \mathbf{T}_{2} & \ddots & \vdots & \mathbf{U}_{2} \\
\vdots & \ddots & \ddots & 0 & \vdots \\
0 & \cdots & 0 & \mathbf{T}_{\mathbf{K}} & \mathbf{U}_{\mathbf{K}} \\
\mathbf{V}_{\mathbf{1}} & \mathbf{V}_{\mathbf{2}} & \ldots & \mathbf{V}_{\mathbf{K}} & \mathbf{E}
\end{array}\right)\left(\begin{array}{c}
\mathbf{J}_{1} \\
\mathbf{J}_{2} \\
\vdots \\
\mathbf{J}_{\mathbf{K}} \\
\overline{\mathbf{J}}
\end{array}\right)=\left(\begin{array}{c}
\mathbf{K}_{\mathbf{1}} \\
\mathbf{K}_{\mathbf{2}} \\
\vdots \\
\mathbf{K}_{\mathbf{K}} \\
\mathbf{Y}
\end{array}\right)
$$

where $\mathbf{E}$ is the negative identity matrix, $\mathbf{Y}$ is the null matrix, and

$$
\left(V_{k}\right)_{i, i^{\prime}}=\frac{\kappa_{k} b_{k}}{\sum_{k^{\prime}=1}^{K} \frac{d B_{k^{\prime}}}{d T} \kappa_{k^{\prime}} b_{k^{\prime}}} \delta_{i, i^{\prime}}
$$

With the addition of the boundary conditions described below, Equation C.37 can now be solved as in the the standard Rybicki method (Rybicki 1971), giving $\mathbf{J}_{\mathbf{k}}$, which can then be used to calculate the temperature correction. 


\section{C.4 Boundary Conditions}

The boundary conditions used for the temperature correction procedure in the present work are the same as those used in a previous work (Z96), where the same temperature correction scheme was used, but with a different discretization method.

At the top of the atmosphere, the boundary condition

$$
\frac{1}{k_{\nu}} \frac{d}{d y}\left(f_{\nu} J_{\nu}\right)=h_{\nu} J_{\nu}
$$

is used, while at the bottom, the condition

$$
J_{\nu}=B_{\nu}
$$

is used.

The boundary condition at the top of the atmosphere can be discretized as

$$
\frac{\left(f_{k} J_{k}\right)\left(y_{2}\right)-\left(f_{k} J_{k}\right)\left(y_{1}\right)}{\frac{1}{2}\left[k_{k}\left(y_{2}\right)+k_{k}\left(y_{1}\right)\right]\left(y_{2}-y_{1}\right)}=\left(h_{k} J_{k}\right)\left(y_{1}\right) \text {. }
$$

Thus, for first order accurate boundary conditions, $\mathbf{A}_{\mathbf{1}}$ is a null matrix and $\mathbf{Q}_{\mathbf{1}}$ is a null vector. Further, the previously unspecified $i=1$ elements of the matrices $\mathbf{T}_{\mathbf{k}}$, and the diagonal matrices $\mathbf{B}_{\mathbf{1}}$ and $\mathbf{C}_{\mathbf{1}}$, are specified as follows:

$$
\begin{aligned}
\left(T_{k}\right)_{1,2} & =\left(C_{1}\right)_{k k}=\frac{-f_{k}\left(y_{2}\right)}{\frac{1}{2}\left(k_{\nu}\left(y_{2}\right)+k_{\nu}\left(y_{1}\right)\right)\left(y_{2}-y_{1}\right)} \\
\left(T_{k}\right)_{1,1} & =\left(B_{1}\right)_{k k}=h_{k}+\frac{f_{k}\left(y_{1}\right)}{\frac{1}{2}\left(k_{\nu}\left(y_{2}\right)+k_{\nu}\left(y_{1}\right)\right)\left(y_{2}-y_{1}\right)}
\end{aligned}
$$

At the bottom of the atmosphere, the boundary condition is trivially discretized, making $\mathbf{C}_{\mathbf{N}}$ a null matrix, while the previously unspecified $i=N_{\text {temp }}$ 
( $\left.N=N_{\text {temp }}\right)$ elements of the $\mathbf{T}_{\mathbf{K}}$ matrices, and the diagonal matrices $\mathbf{B}_{\mathbf{N}}$ and $\mathbf{A}_{\mathbf{N}}$, are specified as follows:

$$
\begin{aligned}
\left(T_{k}\right)_{N, N-1} & =\left(A_{N}\right)_{k k}=0 \\
\left(T_{k}\right)_{N, N} & =\left(B_{N}\right)_{k k}=1 .
\end{aligned}
$$

Finally, the $i=N$ elements of the $\mathbf{K}_{\mathbf{k}}$ vectors, and the $\mathbf{Q}_{\mathbf{N}}$ vector, are given by

$$
\left(K_{k}\right)_{N}=\left(Q_{N}\right)_{k}=B_{k}\left(T\left(y_{N}\right)\right) .
$$




\section{APPENDIX D THE CODE}

This section gives a brief overview of the implementation of the McPHAC code, and the numerical packages and routines that are used. The source-code itself is made available online ${ }^{1}$, and serves as its own documentation. Thus, any questions not answered in the present work should be resolvable by consulting the code.

\section{D.1 Platform and Language}

The McPHAC code is written in the $\mathrm{C}$ language, but makes calls to several Fortran functions written by other groups. The code has been developed and tested on an AMD platform running the Linux operating system, using the GNU gfortran compiler to compile and link both the $\mathrm{C}$ and Fortran code.

\section{D.2 External Routines}

Several external routines are used for standard numerical problems, and these are described here.

\footnotetext{
${ }^{1}$ http://dualcore.physics.mcgill.ca/McPHAC/
} 


\section{D.2.1 LAPACK}

The Linear Algebra PACKage (LAPACK) v3.2.12 routine dgesv() is used to invert full matrices, while the routine $\operatorname{dgtsv}()$ is used to invert tridiagonal matrices. These routines are Fortran routines, and make use of several BLAS routines (see below). The source code for these routines is included with the McPHAC code, but for optimal performance pre-compiled optimized libraries for the appropriate platform should be obtained.

\section{D.2.2 BLAS}

The Basic Linear Algebra Subroutines (BLAS) ${ }^{3}$ are highly optimized linear algebra routines written in Fortran. In addition to any routines called by the above mentioned LAPACK routines, the BLAS routine dgemm() is used for matrix multiplication. The source code for all the BLAS routines are included with the McPHAC code, but it is highly recommended to use pre-compiled optimized libraries for the appropriate platform.

\section{D.2.3 Cubic Spline Interpolation}

Cubic spline interpolation is performed using the Fortran routines spline_cubic_set() and spline_cubic_val(), which are part of the SPLINE library ${ }^{4}$. The code for

\footnotetext{
2 http://www.netlib.org/lapack/

${ }^{3}$ http://www.netlib.org/blas/, accessed 2009 June 20

4 http://people.sc.fsu.edu/ burkardt/f_src/spline/spline.html, accessed 2009 June 21
} 
those two routines, and any routines required by them, are included with the McPHAC code (the library is licenced under the GNU LGPL licence).

\section{D.2.4 Gauss-Legendre Quadrature}

To compute the abscissas and weights for the Gauss-Legendre quadrature, the Fortran routine legendre_com() in the QUADRULE library ${ }^{5}$ is used. That code is licenced under the GNU LGPL licence, and is included with the McPHAC code.

\section{D.2.5 Equation of State}

The equation of state is obtained using OPAL EOS $2001^{6}$ (Rogers et al. 1996) routines, which are written in Fortran. The source code and associated tables are included with the McPHAC code.

\footnotetext{
${ }^{5}$ http://people.sc.fsu.edu/ burkardt/f_src/quadrule/quadrule.html, accessed 2009 June 22

${ }^{6}$ http://www-phys.1lnl.gov/Research/OPAL/opal.html, accessed 2005 June 29
} 


\section{References}

Arnaud, K. A. 1996, in Astronomical Data Analysis Software and Systems V., ed. G. Jacoby \& J. Barnes, Vol. 101 (ASP Conf. Series), 17

Auer, L. 1976, Journal of Quantitative Spectroscopy and Radiative Transfer, 16, 931

Becker, W. et al. 2003, ApJ, 594, 798

Beloborodov, A. M. 2002, ApJL, 566, L85

Boercker, D. B. 1987, ApJL, 316, L95

Bogdanov, S., Rybicki, G. B., \& Grindlay, J. E. 2007, ApJ, 670, 668

Brown, E. F., Bildsten, L., \& Rutledge, R. E. 1998, ApJL, 504, L95

Brown, E. F. \& Cumming, A. 2009, ApJ, 698, 1020

Burwitz, V., Zavlin, V. E., Neuhäuser, R., Predehl, P., Trümper, J., \& Brinkman, A. C. 2001, A\&A, 379, L35

Cackett, E. M., Wijnands, R., Linares, M., Miller, J. M., Homan, J., \& Lewin, W. H. G. 2006, M.N.R.A.S., 372, 479

Cackett, E. M., Wijnands, R., Miller, J. M., Brown, E. F., \& Degenaar, N. 2008, ApJL, 687, L87

Campana, S., Israel, G. L., Stella, L., Gastaldello, F., \& Mereghetti, S. 2004, ApJ, 601,474

Cornelisse, R., Wijnands, R., \& Homan, J. 2007, M.N.R.A.S., 380, 1637 
Degenaar, N. et al. 2009, M.N.R.A.S., 396, L26

Feautrier, P. 1964, Comptes Rendus Academie des Sciences (serie non specifiee), 258,3189

Gänsicke, B. T., Braje, T. M., \& Romani, R. W. 2002, A\&A, 386, 1001

Gendre, B., Barret, D., \& Webb, N. 2003a, A\&A, 403, L11

Gendre, B., Barret, D., \& Webb, N. A. 2003b, A\&A, 400, 521

Grindlay, J. E., Heinke, C. O., Edmonds, P. D., Murray, S. S., \& Cool, A. M. 2001, ApJL, 563, L53

Guillot, S., Rutledge, R. E., Bildsten, L., Brown, E. F., Pavlov, G. G., \& Zavlin, V. E. 2009a, M.N.R.A.S., 392, 665

Guillot, S., Rutledge, R. E., Brown, E. F., Pavlov, G. G., \& Zavlin, V. E. 2009b, ApJ, 699, 1418

Haensel, P. \& Zdunik, J. L. 1990, A\&A, 227, 431

- 2008, A\&A, 480, 459

Heinke, C. O., Grindlay, J. E., Edmonds, P. D., Lloyd, D. A., Murray, S. S., Cohn, H. N., \& Lugger, P. M. 2003a, ApJ, 598, 516

Heinke, C. O., Grindlay, J. E., Lloyd, D. A., \& Edmonds, P. D. 2003b, ApJ, 588, 452

Heinke, C. O., Jonker, P. G., Wijnands, R., \& Taam, R. E. 2007, ApJ, 660, 1424

Heinke, C. O., Rybicki, G. B., Narayan, R., \& Grindlay, J. E. 2006, ApJ, 644, 1090

Hummer, D. G. 1962, M.N.R.A.S., 125, 21

Iglesias, C. A. \& Rogers, F. J. 1996, ApJ, 464, 943

Iglesias, C. A. \& Rose, S. J. 1996, ApJL, 466, L115+ 
in’t Zand, J. J. M. 2005, A\&A, 441, L1

in’t Zand, J. J. M., van Kerkwijk, M. H., Pooley, D., Verbunt, F., Wijnands, R., \&

Lewin, W. H. G. 2001, ApJL, 563, L41

Jonker, P. G., Bassa, C. G., \& Wachter, S. 2007a, M.N.R.A.S., 377, 1295

Jonker, P. G., Galloway, D. K., McClintock, J. E., Buxton, M., Garcia, M., \&

Murray, S. 2004, M.N.R.A.S., 354, 666

Jonker, P. G., Steeghs, D., Chakrabarty, D., \& Juett, A. M. 2007b, ApJL, 665, L147

Karzas, W. J. \& Latter, R. 1961, ApJ Supp., 6, 167

Lattimer, J. M. \& Prakash, M. 2001, ApJ, 550, 426

— 2007, Phys. Rep., 442, 109

Lloyd, D. A. 2003, PhD thesis, AA(HARVARD UNIVERSITY)

Lugger, P. M., Cohn, H. N., Heinke, C. O., Grindlay, J. E., \& Edmonds, P. D. 2007, ApJ, 657, 286

McClintock, J. E., Narayan, R., \& Rybicki, G. B. 2004, ApJ, 615, 402

Mihalas, D. 1970, Stellar atmospheres (W. H. Freeman and Company, San Francisco)

— 1978, Stellar atmospheres /2nd edition/ (San Francisco, W. H. Freeman and Co., 1978. 650 p.)

Misner, C. W., Thorne, K. S., \& Wheeler, J. A. 1973, Gravitation (W. H. Freeman)

Ogley, R. N., Chaty, S., Crocker, M., Eyres, S. P. S., Kenworthy, M. A., Richards, A. M. S., Rodríguez, L. F., \& Stirling, A. M. 2002, M.N.R.A.S., 330, 772 
Pavlov, G. G., Zavlin, V. E., Truemper, J., \& Neuhaeuser, R. 1996, ApJL, 472, L33

Pechenick, K. R., Ftaclas, C., \& Cohen, J. M. 1983, ApJ, 274, 846

Pons, J. A., Walter, F. M., Lattimer, J. M., Prakash, M., Neuhäuser, R., \& An, P. 2002, ApJ, 564, 981

Rajagopal, M. \& Romani, R. W. 1996, ApJ, 461, 327

Rogers, F. J., Swenson, F. J., \& Iglesias, C. A. 1996, ApJ, 456, 902

Rutledge, R. E., Bildsten, L., Brown, E. F., Pavlov, G. G., \& Zavlin, V. E. 1999, ApJ, 514, 945

- 2000, ApJ, 529, 985

— 2001a, ApJ, 559, 1054

— 2001b, ApJ, 551, 921

- 2002a, ApJ, 578, 405

— 2002b, ApJ, 577, 346

Rutledge, R. E., Bildsten, L., Brown, E. F., Pavlov, G. G., Zavlin, V. E., \& Ushomirsky, G. 2002c, ApJ, 580, 413

Rybicki, G. B. 1971, Journal of Quantitative Spectroscopy and Radiative Transfer, 11,589

Rybicki, G. B. \& Lightman, A. P. 1979, Radiative processes in astrophysics (Wiley)

Shevchuk, A. S. H., Fox, D. B., \& Rutledge, R. E. 2009, ArXiv e-prints

Shternin, P. S., Yakovlev, D. G., Haensel, P., \& Potekhin, A. Y. 2007, M.N.R.A.S., 382, L43 
Sutherland, R. S. 1998, M.N.R.A.S., 300, 321

Tomsick, J. A., Gelino, D. M., Halpern, J. P., \& Kaaret, P. 2004, ApJ, 610, 933

Tomsick, J. A., Gelino, D. M., \& Kaaret, P. 2005, ApJ, 635, 1233

— 2007, ApJ, 663, 461

Ushomirsky, G. \& Rutledge, R. E. 2001, M.N.R.A.S., 325

Webb, N. A. \& Barret, D. 2007, ApJ, 671, 727

Webb, N. A., Wheatley, P. J., \& Barret, D. 2006, A\&A, 445, 155

Weiner, B. et al. 2009, Astronomy, 2010, 61P

Wijnands, R., Heinke, C. O., \& Grindlay, J. E. 2002, ApJ, 572, 1002

Wijnands, R., Nowak, M., Miller, J. M., Homan, J., Wachter, S., \& Lewin, W. H. G. 2003, ApJ, 594, 952

Yakovlev, D. G., Levenfish, K. P., \& Haensel, P. 2003, A\&A, 407, 265

Zavlin, V. E. 2006, ApJ, 638, 951

Zavlin, V. E. \& Pavlov, G. G. 1998, A\&A, 329, 583

Zavlin, V. E., Pavlov, G. G., Sanwal, D., Manchester, R. N., Trümper, J., Halpern, J. P., \& Becker, W. 2002, ApJ, 569, 894

Zavlin, V. E., Pavlov, G. G., \& Shibanov, Y. A. 1994, Astronomical and Astrophysical Transactions, 4, 307

- 1996, A\&A, 315, 141

Zavlin, V. E., Pavlov, G. G., \& Trumper, J. 1998, A\&A, 331, 821 